\title{
A magnetic version of the Smilansky-Solomyak model
}

\author{
Diana Barseghyan \\ Department of Mathematics, University of Ostrava, 30. dubna 22, 70103 Ostrava, \\ Czech Republic \\ Nuclear Physics Institute, Academy of Sciences of the Czech Republic, Hlavní 130, \\ 25068 Řež near Prague, Czech Republic \\ E-mail: dianabar@ujf.cas.cz, diana.barseghyan@osu.cz \\ Pavel Exner \\ Nuclear Physics Institute, Academy of Sciences of the Czech Republic, Hlavní 130, \\ 25068 Řež near Prague, Czech Republic \\ Doppler Institute, Czech Technical University, Břehová 7, 11519 Prague, Czech \\ Republic \\ E-mail: exner@ujf.cas.cz
}

\begin{abstract}
We analyze spectral properties of two mutually related families of magnetic Schrödinger operators, $H_{\mathrm{Sm}}(A)=(i \nabla+A)^{2}+\omega^{2} y^{2}+\lambda y \delta(x)$ and $H(A)=$ $(i \nabla+A)^{2}+\omega^{2} y^{2}+\lambda y^{2} V(x y)$ in $L^{2}\left(\mathbb{R}^{2}\right)$, with the parameters $\omega>0$ and $\lambda<0$, where $A$ is a vector potential corresponding to a homogeneous magnetic field perpendicular to the plane and $V$ is a regular nonnegative and compactly supported potential. We show that the spectral properties of the operators depend crucially on the one-dimensional Schrödinger operators $L=-\frac{\mathrm{d}^{2}}{\mathrm{~d} x^{2}}+\omega^{2}+\lambda \delta(x)$ and $L(V)=-\frac{\mathrm{d}^{2}}{\mathrm{~d} x^{2}}+\omega^{2}+\lambda V(x)$, respectively. Depending on whether the operators $L$ and $L(V)$ are positive or not, the spectrum of $H_{\mathrm{Sm}}(A)$ and $H(V)$ exhibits a sharp transition.
\end{abstract}

Keywords: Discrete spectrum, essential spectrum, Smilansky-Solomyak model, spectral transition, homogeneous magnetic field

Submitted to: J. Phys. A.: Math. Theor. 


\section{Introduction}

Irreversible dynamics of quantum systems is usually described through a coupling of the object, regarded as an open or unstable system, to another one that plays the role of a 'heat bath'. The latter is usually supposed to be a 'large' system having an infinite numbers of degrees of freedom and the Hamiltonian with a continuous spectrum, moreover, the presence (or absence) of irreversible modes is determined by the energies involved rather than the coupling strength between the object and the bath. While this is all true in many cases, it need not be true in general. This was demonstrated by Uzy Smilansky using a simple model Sm04 which was subsequently analyzed in detail and generalized by Mikhail Solomyak and coauthors [So04a, So04b, ES05a, ES05b, So06a, So06b, NS06, RS07, see also [Gu11] and [ELT17].

In the simplest case the model is described by the Hamiltonian

$$
H_{\mathrm{Sm}}=-\frac{\partial^{2}}{\partial x^{2}}+\frac{1}{2}\left(-\frac{\partial^{2}}{\partial y^{2}}+y^{2}\right)+\lambda y \delta(x)
$$

in $L^{2}\left(\mathbb{R}^{2}\right)$ with the natural domain and exhibits a transition between two types of spectral behavior: for $|\lambda| \leq \sqrt{2}$ the operator (1.1) is bounded from below, while for $|\lambda|>\sqrt{2}$ its spectrum fills the real line [So04a]. The factor $\frac{1}{2}$ is not important and can be changed by a scaling of one of the variables. If we replace it by one, for instance, the critical value of the coupling constant will be $\lambda=2$. The transition between the two regimes can be interpreted also dynamically [Gu11]: in the supercritical regime the $y$-dependent binding energy of $\delta$ interaction wins over the oscillator potential and the wave packet can escape to infinity along the singular channel.

While mathematically we deal with the same object, from the physical point of view one can interpret it in two different ways. In the original Smilansky paper Sm04] it was meant as a system of two one dimensional components, a particle motion on a line to which a heat bath consisting of a single harmonic oscillator is coupled in a coordinate dependent way. In the generalizations mentioned above the line was replaced by other simple configuration spaces, a loop (in other words, segment with periodic boundary conditions) or a graph, and the bath could be anharmonic or multidimensional (but still with a finite number of degrees of freedom).

Another point of view, which can be associated with the work of Solomyak and coauthors, is to associate the Hamiltonian (1.1) with a two-dimensional system in which the particle moves in the potential which is the sum of the oscillator 'channel' and the singular component with the position-dependent coupling strength. Viewed from this angle, the system brings to mind motion in a potential with channels which are below unbounded and narrowing towards infinity. In this situation one may also observe a jump transition from a below bounded to below unbounded spectrum as was first noted in [Zn98, a class of models of this type was analyzed recently in [EB12, BEKT16]. The analogy becomes even more convincing when we recall that the model (1.1) has a 'regular' analogue [BE14, BE17] in which the $\delta$ interaction is replaced by a nonsingular potential properly scaled. In this case, of course, the 'first', two subsystem, 
interpretation is lost unless we try to interpret the potential as a sort of nonlocal positiondependent subsystem coupling.

The main question we want to address in the present paper is what will happen with the model in its two-dimensional version when the particle it describes is charged and exposed to a homogeneous magnetic field perpendicular to the plane, in other words, what are the properties of the operator

$$
H_{\mathrm{Sm}}(A)=(i \nabla+A)^{2}+\omega^{2} y^{2}+\lambda y \delta(x)
$$

with $\lambda \in \mathbb{R}$ and $\omega>0$, where the vector potential $A$ corresponds to the indicated magnetic field of intensity $B>0$. We may consider non-positive $\lambda$ only; as in the nonmagnetic case this is due to mirror symmetry but the argument is a bit trickier. The magnetic field changes direction when observed in a mirror, however, switching the sign of both the variables we return to the original $A$ and at the same time the last term on the right-hand side of (1.2) changes sign. It is clear that now the two-subsystem interpretation is ultimately lost, therefore it is appropriate to speak of (1.2) as of the Hamiltonian of the magnetic Smilansky-Solomyak model.

The dynamics of the model combines the influence of several forces and its properties are not a priori obvious. For $\lambda=0$ the spectrum is absolutely continuous and the particle moves along the parabolic channel provided its energy is larger than $\sqrt{\omega^{2}+B^{2}}$, and moreover, the transport is stable against localized perturbations [EK00]. If both $\omega$ and $\lambda$ vanish, operator (1.2) is the Landau Hamiltonian the spectrum of which is known to be pure point, consisting of the Landau levels $(2 n+1) B, n=0,1,2, \ldots$. Was the singular term position independent, just $\lambda \delta(x)$, it would make the spectrum absolutely continuous corresponding to transport in the $y$ direction as one could check in a way similar to the Iwatsuka model [CFKS87, Sec. 6.5], [EK15, Sec. 7.2.3], or to magnetic transport along a barrier [FGW00]. The operator (1.2) with $\omega=0$ has not been analyzed to the best of our knowledge, but one can expect that it will exhibit some transport properties again; at least we will show, as a byproduct of our results here, that its spectrum covers the whole real axis whenever $\lambda \neq 0$.

The oscillator potential, however, acts against a transport in the $y$ direction. We are going to show that the resulting behavior is determined by the balance of the two forces, in a way to a large degree similar to the nonmagnetic case, $A=0$. To be specific, we introduce the comparison operator,

$$
L=-\frac{\mathrm{d}^{2}}{\mathrm{~d} x^{2}}+\omega^{2}+\lambda \delta(x)
$$

on $L^{2}(\mathbb{R})$ with the usual domain [AGHH05; our goal is to establish a correspondence between the spectral regime of $H_{\mathrm{Sm}}(A)$ and the positivity of the operator (1.3). We are going to show that the spectrum is bounded from below provided $\inf \sigma(L)>0$ - we speak here about the subcritical case - and it has a purely discrete character below $\sqrt{\omega^{2}+B^{2}}$ being nonempty whenever $\lambda \neq 0$. In the critical case, inf $\sigma(L)=0$, the operator $H_{\mathrm{Sm}}(A)$ remains positive but its spectrum is purely essential and equal 
to $[0, \infty)$. Finally, if one passes to the supercritical regime, $\inf \sigma(L)<0$, an abrupt transition occurs and the spectrum fills now the whole real line.

In a similar way, the 'regular' version of the model [BE14, BE17] mentioned above has also its magnetic counterpart. The dynamics is this case described by the Hamiltonian

$$
H(A)=(i \nabla+A)^{2}+\omega^{2} y^{2}+\lambda y^{2} V(x y),
$$

where $V$ is a nonnegative, sufficiently smooth function with $\operatorname{supp}(V) \subset\left[-s_{0}, s_{0}\right]$ for some $s_{0}>0$, furthermore, $\lambda \leq 0$ and $\omega>0$, and the magnetic potential $A$ corresponds as before to a homogeneous magnetic field of the intensity $B>0$. Note that the analogy is not complete because both parts of the scalar potential are mirror symmetric with respect to the $x$ axis, however, the effect which we are interested in depends on the presence of an attractive interaction in the $y$ direction, irrespective whether is one- or two-sided. Spectral properties of the operator (1.4) will be the topic of the second part of the paper. The abrupt spectral transition occurs here again. The comparison operator will be

$$
L(V)=-\frac{\mathrm{d}^{2}}{\mathrm{~d} x^{2}}+\omega^{2}+\lambda V(x)
$$

on $L^{2}(\mathbb{R})$ with the domain $\mathcal{H}^{2}(\mathbb{R})$, and its spectral threshold will be shown to be decisive: $H(A)$ will be bounded from below provided if $L(V)$ is nonnegative, and its spectrum will fill the whole real line in the opposite case.

\section{Spectrum of $H_{\mathrm{Sm}}(A)$}

Before coming to our proper subject we note that in order to interpret $H_{\mathrm{Sm}}(A)$ as a quantum mechanical Hamiltonian, one has check its self-adjointness. In the subcritical case, when $L$ is strictly positive, we can consider first the domain $\mathcal{D}_{0}$ consisting of the family of functions $v$ twice differentiable except at the $y$ axis, $x=0$, continuous there and satisfying the matching conditions $\frac{\partial v}{\partial x}(0+, y)-\frac{\partial v}{\partial x}(0-, y)=\lambda y v(0, y)$ and to identify $H_{\mathrm{Sm}}(A)$ with the Friedrichs extension of such an operator. In other words, we will consider the quadratic form

$$
Q\left(H_{\mathrm{Sm}}(A)\right)[u]=\int_{\mathbb{R}^{2}}\left[\left|i \frac{\partial u}{\partial x}-B y u\right|^{2}+\left|\frac{\partial u}{\partial y}\right|^{2}+\omega^{2} y^{2}|u|^{2}\right] \mathrm{d} x \mathrm{~d} y+\lambda \int_{\mathbb{R}} y|u(0, y)|^{2} \mathrm{~d} y
$$

and demonstrate that it is closed on $\mathcal{D}:=\mathcal{H}^{1}\left(\mathbb{R}^{2}\right) \cap\left\{\int_{\mathbb{R}^{2}} y^{2}|u(x, y)|^{2} \mathrm{~d} x \mathrm{~d} y<\infty\right\}$ and bounded from below provided $\inf \sigma(L)>0$, and therefore associated with a unique self-adjoint operator.

The approach based on quadratic forms fails, of course, if we cannot ensure that the operator is bounded from below. To make things simple, we can bypass this trouble by noting that $H_{\mathrm{Sm}}(A)$ is essentially self-adjoint on $\mathcal{D}_{0}$. Indeed, it is easy to check that such a operator is densely defined and symmetric. To ensure that its deficiency indices coincide, it is by [GMNT16, Thm. 2.8] sufficient to check that its commutes with a conjugation, i.e. an antilinear map $L^{2}\left(\mathbb{R}^{2}\right) \rightarrow L^{2}\left(\mathbb{R}^{2}\right)$ which is norm preserving 
and idempotent; choosing $\mathcal{C}:(\mathcal{C} v)(x, y)=\overline{u(-x, y)}$ we get the claim. Then we know that $H_{\mathrm{Sm}}(A) \uparrow D_{0}$ has self-adjoint extension and will show that the indicated spectral properties hold for any such extension.

\subsection{The subcritical case}

As we have indicated, to establish the self-adjointness of $H_{\mathrm{Sm}}(A)$ one has to check that the form (2.1) is bounded from below and closed if inf $\sigma(L)=\omega^{2}-\frac{1}{4} \lambda^{2}>0$. First we will show that the operator is in fact positive even if the last inequality is not sharp.

Proposition 2.1. Let $\lambda \geq-2 \omega$, then $H_{\mathrm{Sm}}(A) \uparrow \mathcal{D}_{0} \geq 0$.

Proof. For every $u \in \mathcal{D}_{0}$ the form (2.1) can be estimated form below by neglecting the 'transverse' contribution to the kinetic energy

$$
Q\left(H_{\mathrm{Sm}}(A)\right)[u] \geq \int_{\mathbb{R}^{2}}\left[\left|i \frac{\partial u}{\partial x}-B y u\right|^{2}+\omega^{2} y^{2}|u|^{2}\right] \mathrm{d} x \mathrm{~d} y+\lambda \int_{\mathbb{R}} y|u(0, y)|^{2} \mathrm{~d} y .
$$

For any fixed $y$ the form $u(\cdot, y) \mapsto \int_{\mathbb{R}}\left|i \frac{\partial u}{\partial x}-B y u\right|^{2}+\omega^{2} y^{2}|u|^{2} \mathrm{~d} x+\lambda y|u(0, y)|^{2}$ corresponds to the essentially self-adjoint operator

$$
\left(i \frac{\mathrm{d}}{\mathrm{d} x}-B y\right)^{2}+\omega^{2} y^{2}+\lambda y \delta(x)
$$

whose closure has $\mathcal{H}^{1}(\mathbb{R})$ as its form domain. This operator is unitarily equivalent to $y^{2} L$ which is positive by assumption if $y>0$, and to $y^{2} \widetilde{L} \geq 0$ if $y<0$, where

$$
\widetilde{L}:=-\frac{d^{2}}{d x^{2}}+\omega^{2}-\lambda \delta(x) ;
$$

this establishes the sought claim.

Proposition 2.2. The form (2.1) is closed if $\lambda>-2 \omega$.

Proof. Let $\left\{u_{n}\right\}_{n=1}^{\infty} \subset \mathcal{D}$ be a sequence converging to some $u \in L^{2}\left(\mathbb{R}^{2}\right)$ and satisfying

$$
Q\left(H_{\mathrm{Sm}}\right)(A)\left[u_{n}-u_{m}\right] \rightarrow 0
$$

as $m, n \rightarrow \infty$. By the assumption one can choose $\alpha \in\left(\frac{|\lambda|}{2 \omega}, 1\right)$ and rewrite the form value in question as

$$
\begin{aligned}
& Q\left(H_{\mathrm{Sm}}(A)\right)\left[u_{n}-u_{m}\right]=(1-\alpha)\left(\int_{\mathbb{R}^{2}}\left|(i \nabla+A)\left(u_{n}-u_{m}\right)\right|^{2} \mathrm{~d} x \mathrm{~d} y\right. \\
& \left.\quad+\omega^{2} \int_{\mathbb{R}^{2}} y^{2}\left|u_{n}-u_{m}\right|^{2} \mathrm{~d} x \mathrm{~d} y\right)+\alpha\left(\int_{\mathbb{R}^{2}}\left|(i \nabla+A)\left(u_{n}-u_{m}\right)\right|^{2} \mathrm{~d} x \mathrm{~d} y\right. \\
& \left.\quad+\omega^{2} \int_{\mathbb{R}^{2}} y^{2}\left|u_{n}-u_{m}\right|^{2} \mathrm{~d} x \mathrm{~d} y+\frac{\lambda}{\alpha} \int_{\mathbb{R}} y\left|u_{n}(0, y)-u_{m}(0, y)\right|^{2} \mathrm{~d} x\right) .
\end{aligned}
$$

In the same way as in the proof of the previous proposition one can check that the second summand on the right-hand side of (2.4) is nonnegative, hence neglecting it we 
estimate $Q\left(H_{\mathrm{Sm}}(A)\right)\left[u_{n}-u_{m}\right]$ from below by the first summand which in view of (2.3) tends to zero as $m, n \rightarrow \infty$. Since $\alpha \neq 1$ by construction, this means that the sequence $\left\{Q_{0}(A)\left[u_{n}\right]\right\}$ is Cauchy, where $Q_{0}(A)$ is the 'unperturbed' form

$$
u \mapsto Q_{0}(A)[u]:=\int_{\mathbb{R}^{2}}\left[|(i \nabla+A) u|^{2}+\omega^{2} y^{2}|u|^{2}\right] \mathrm{d} x \mathrm{~d} y
$$

defined on $\mathcal{D}$. It is not difficult to verify that $Q_{0}(A)$ is closed and this in turn implies that the limit function $u$ belongs to $\mathcal{D}$ and

$$
\int_{\mathbb{R}^{2}}\left[\left|(i \nabla+A)\left(u_{n}-u\right)\right|^{2}+\omega^{2} y^{2}\left|u_{n}-u\right|^{2}\right] \mathrm{d} x \mathrm{~d} y \rightarrow 0 \quad \text { as } \quad n \rightarrow \infty
$$

cf. [RS80, Problem VIII.15]. It remains to check that $\int_{\mathbb{R}} y\left|u_{n}(0, y)-u(0, y)\right|^{2} \mathrm{~d} y \rightarrow 0$ holds as well. Using a couple of simple estimates,

$$
\begin{aligned}
& \int_{\mathbb{R}}|y||v(0, y)|^{2} \mathrm{~d} y \leq \frac{2}{\omega} \int_{\mathbb{R}^{2}}\left(\left|\frac{\partial v}{\partial x}\right|^{2}+\omega^{2} y^{2}|v(x, y)|^{2}\right) \mathrm{d} x \mathrm{~d} y \\
& \leq \frac{1}{2 \omega} \int_{\mathbb{R}^{2}}\left(\left|\frac{\partial v}{\partial x}\right|^{2}+\left|\frac{\partial v}{\partial y}+i B x v\right|^{2}(x, y)+\omega^{2} y^{2}|v(x, y)|^{2}\right) \mathrm{d} x \mathrm{~d} y \\
& =\frac{1}{2 \omega} Q_{0}(a)[v]
\end{aligned}
$$

and inserting $v=u_{n}-u$, we conclude the proof.

This guarantees that in the subcritical case there is a unique self-adjoint operator $H_{\mathrm{Sm}}(A)$ associated with the form (2.1).

\subsection{The essential spectrum}

In fact will first show that the essential spectrum is nonempty independently of $\lambda$.

Theorem 2.1. $\sigma_{\mathrm{ess}}\left(H_{\mathrm{Sm}}(A)\right) \supset\left[\sqrt{\omega^{2}+B^{2}}, \infty\right)$.

Proof. It is sufficient to construct a Weyl sequence for any number $\mu>\sqrt{\omega^{2}+B^{2}}$. To this aim, we fix first a positive number $\varepsilon$ and construct a function $\phi$ such that

$$
\left\|H_{\mathrm{Sm}}(A) \phi-\mu \phi\right\|_{L^{2}\left(\mathbb{R}^{2}\right)}<\varepsilon\|\phi\|
$$

We employ the functions

$$
\begin{aligned}
& \varphi_{k, \alpha, m}(x, y):= \\
& \frac{1}{\sqrt{2 \pi \operatorname{vol}(E)}}\left(\int_{E} g\left(y-\frac{\xi B}{\omega^{2}+B^{2}}\right) e^{i \xi(x-\alpha k)} \mathrm{d} \xi\right) \eta\left(\frac{x}{k}\right) \chi\left(\frac{y}{k}\right),
\end{aligned}
$$

where $g$ is the normalized eigenfunction associated with the principal eigenvalue of the harmonic oscillator modified by the presence of the magnetic field, $h_{\mathrm{osc}}=-\frac{\mathrm{d}^{2}}{\mathrm{~d} y^{2}}+$ 
A magnetic version of the Smilansky-Solomyak model

$\left(\omega^{2}+B^{2}\right) y^{2}$ on $L^{2}(\mathbb{R})$, the functions $\eta \in C_{0}^{\infty}(1, m), \chi \in C_{0}^{\infty}(-1,1)$ are supposed to satisfy the following requirements,

$$
\eta(z) \geq \frac{1}{2} \quad \text { if } \quad z \in\left(\frac{3}{2}, \frac{m}{2}\right), \quad \chi(z) \geq \frac{1}{2} \quad \text { if } \quad z \in\left(-\frac{1}{2}, \frac{1}{2}\right),
$$

and the set $E$ is defined by

$$
E=\left(\delta_{1}(\varepsilon), \delta_{2}(\varepsilon)\right):=\left\{\xi: \frac{\sqrt{(\tilde{\mu}-\varepsilon)\left(\omega^{2}+B^{2}\right)}}{\omega}<\xi<\frac{\sqrt{(\tilde{\mu}+\varepsilon)\left(\omega^{2}+B^{2}\right)}}{\omega}\right\},
$$

where $\tilde{\mu}:=\mu-\sqrt{\omega^{2}+B^{2}}$ and $k, m, \alpha \in \mathbb{N}$ are positive integers to be chosen later. Note that $\operatorname{supp}\left(\psi_{k, \alpha, m}\right) \subset[k, m k] \times[-k, k]$, and therefore

$$
\frac{\partial \psi_{k, \alpha, m}}{\partial x}(0+, y)=\frac{\partial \psi_{k, \alpha, m}}{\partial x}(0-, y)=\lambda y \psi_{k, \alpha, m}(0, y)=0
$$

which means that the functions $\psi_{k, \alpha, m}$ belong to the domain of $H_{\mathrm{Sm}}(A)$ as needed.

First we observe that $\left\|\varphi_{k, \alpha, m}\right\|_{L^{2}\left(\mathbb{R}^{2}\right)} \geq \frac{1}{8}$ because

$$
\begin{aligned}
& \int_{\mathbb{R}^{2}}\left|\varphi_{k, \alpha, m}(x, y)\right|^{2} \mathrm{~d} x \mathrm{~d} y \\
& =\frac{1}{2 \pi \operatorname{vol}(E)} \int_{\mathbb{R}^{2}}\left|\int_{E} g\left(y-\frac{\xi B}{\omega^{2}+B^{2}}\right) e^{i \xi(x-\alpha k)} \mathrm{d} \xi\right|^{2} \eta^{2}\left(\frac{x}{k}\right) \chi^{2}\left(\frac{y}{k}\right) \mathrm{d} x \mathrm{~d} y \\
& =\frac{1}{2 \pi \operatorname{vol}(E)} \int_{k}^{m k} \int_{-k}^{k}\left|\int_{E} g\left(y-\frac{\xi B}{\omega^{2}+B^{2}}\right) e^{i \xi(x-\alpha k)} \mathrm{d} \xi\right|^{2} \eta^{2}\left(\frac{x}{k}\right) \chi^{2}\left(\frac{y}{k}\right) \mathrm{d} x \mathrm{~d} y \\
& \quad \geq \frac{1}{32 \pi \operatorname{vol}(E)} \int_{-k / 2}^{k / 2} \int_{3 k / 2}^{m k / 2}\left|\int_{E} g\left(y-\frac{\xi B}{\omega^{2}+B^{2}}\right) e^{i \xi(x-\alpha k)} \mathrm{d} \xi\right|^{2} \mathrm{~d} y \mathrm{~d} x \\
& =\frac{1}{32 \pi \operatorname{vol}(E)} \int_{-k / 2}^{k / 2} \int_{(3-2 \alpha) k / 2}^{(m-2 \alpha) k / 2}\left|\int_{E} g\left(y-\frac{\xi B}{\omega^{2}+B^{2}}\right) e^{i \xi x} \mathrm{~d} \xi\right|^{2} \mathrm{~d} y \mathrm{~d} x .
\end{aligned}
$$

By choosing $\alpha=\alpha(k)$ and $m=m(\alpha, k)$ large enough one is able to guarantee that for every $y \in\left(-\frac{k}{2}, \frac{k}{2}\right)$ we have

$$
\begin{aligned}
& \frac{1}{2 \pi} \int_{(3-2 \alpha) k / 2}^{(m-2 \alpha) k / 2}\left|\int_{E} g\left(y-\frac{\xi B}{\omega^{2}+B^{2}}\right) e^{i \xi x} \mathrm{~d} \xi\right|^{2} \mathrm{~d} x \\
& \quad \geq \frac{1}{4 \pi} \int_{\mathbb{R}}\left|\int_{E} g\left(y-\frac{\xi B}{\omega^{2}+B^{2}}\right) e^{i \xi x} \mathrm{~d} \xi\right|^{2} \mathrm{~d} x \\
& \quad=\frac{1}{2} \int_{E} g^{2}\left(y-\frac{x B}{\omega^{2}+B^{2}}\right) \mathrm{d} x,
\end{aligned}
$$

where in the last step we have employed Plancherel formula. This estimate together 
A magnetic version of the Smilansky-Solomyak model

with (2.7) gives for large $k$ the inequalities

$$
\begin{aligned}
& \int_{\mathbb{R}^{2}}\left|\varphi_{k, \alpha, m}(x, y)\right|^{2} \mathrm{~d} x \mathrm{~d} y \geq \frac{1}{32 \operatorname{vol}(E)} \int_{-k / 2}^{k / 2} \int_{E}\left|g\left(y-\frac{x B}{\omega^{2}+B^{2}}\right)\right|^{2} \mathrm{~d} x \mathrm{~d} y \\
& \geq \frac{1}{32 \operatorname{vol}(E)} \int_{E} \int_{-k / 2-x B /\left(\omega^{2}+B^{2}\right)}^{k / 2-x B /\left(\omega^{2}+B^{2}\right)} g^{2}(z) \mathrm{d} z \mathrm{~d} x \\
& \quad \geq \frac{1}{64} \int_{\mathbb{R}}|g(z)|^{2} \mathrm{~d} z=\frac{1}{64} .
\end{aligned}
$$

Our next aim is to show the validity of (2.5) with an appropriate choice of $k, \alpha(k)$ and $m(\alpha, k)$. By a straightforward calculation one gets

$$
\begin{aligned}
& \sqrt{2 \pi} \frac{\partial^{2} \varphi_{k, \alpha, m}}{\partial y^{2}}=\frac{1}{\sqrt{\operatorname{vol}(E)}}\left(\int_{E} g^{\prime \prime}\left(y-\frac{\xi B}{\omega^{2}+B^{2}}\right) e^{i \xi(x-\alpha k)} \mathrm{d} \xi\right) \eta\left(\frac{x}{k}\right) \chi\left(\frac{y}{k}\right) \\
& +\frac{2}{k \sqrt{\operatorname{vol}(E)}}\left(\int_{E} g^{\prime}\left(y-\frac{\xi B}{\omega^{2}+B^{2}}\right) e^{i \xi(x-\alpha k)} \mathrm{d} \xi\right) \eta\left(\frac{x}{k}\right) \chi^{\prime}\left(\frac{y}{k}\right) \\
& +\frac{1}{k^{2} \sqrt{\operatorname{vol}(E)}}\left(\int_{E} g\left(y-\frac{\xi B}{\omega^{2}+B^{2}}\right) e^{i \xi(x-\alpha k)} \mathrm{d} \xi\right) \eta\left(\frac{x}{k}\right) \chi^{\prime \prime}\left(\frac{y}{k}\right),
\end{aligned}
$$

and

$$
\begin{aligned}
& \sqrt{2 \pi} \frac{\partial^{2} \varphi_{k, \alpha, m}}{\partial x^{2}}=-\frac{1}{\sqrt{\operatorname{vol}(E)}}\left(\int_{E} \xi^{2} g\left(y-\frac{\xi B}{\omega^{2}+B^{2}}\right) e^{i \xi(x-\alpha k)} \mathrm{d} \xi\right) \eta\left(\frac{x}{k}\right) \chi\left(\frac{y}{k}\right) \\
& +\frac{2 i}{k \sqrt{\operatorname{vol}(E)}}\left(\int_{E} \xi g\left(y-\frac{\xi B}{\omega^{2}+B^{2}}\right) e^{i \xi(x-\alpha k)} \mathrm{d} \xi\right) \eta^{\prime}\left(\frac{x}{k}\right) \chi\left(\frac{y}{k}\right) \\
& +\frac{1}{k^{2} \sqrt{\operatorname{vol}(E)}}\left(\int_{E} g\left(y-\frac{\xi B}{\omega^{2}+B^{2}}\right) e^{i \xi(x-\alpha k)} \mathrm{d} \xi\right) \eta^{\prime \prime}\left(\frac{x}{k}\right) \chi\left(\frac{y}{k}\right), \\
& \sqrt{2 \pi} y \frac{\partial \varphi_{k, \alpha, m}}{\partial x}=\frac{i y}{\sqrt{\operatorname{vol}(E)}}\left(\int_{E} \xi g\left(y-\frac{\xi B}{\omega^{2}+B^{2}}\right) e^{i \xi(x-\alpha k)} \mathrm{d} \xi\right) \eta\left(\frac{x}{k}\right) \chi\left(\frac{y}{k}\right) \\
& +\frac{y}{k \sqrt{\operatorname{vol}(E)}}\left(\int_{E} g\left(y-\frac{\xi B}{\omega^{2}+B^{2}}\right) e^{i \xi(x-\alpha k} \mathrm{d} \xi\right) \eta^{\prime}\left(\frac{x}{k}\right) \chi\left(\frac{y}{k}\right) .
\end{aligned}
$$

We want to show that choosing $k$ sufficiently large one can make the last two terms on the right-hand side of the first equation (2.8) as small as one wishes in the $L^{2}$ norm, and the same for the last two terms of the second equation (2.9) and the last term of (2.10). This follows from the following estimates,

$$
\begin{aligned}
& \frac{1}{k^{2} \operatorname{vol}(E)} \int_{\mathbb{R}^{2}}\left|\int_{E} g^{\prime}\left(y-\frac{\xi B}{\omega^{2}+B^{2}}\right) e^{i \xi(x-\alpha k)} \mathrm{d} \xi\right|^{2} \eta^{2}\left(\frac{x}{k}\right)\left(\chi^{\prime}\right)^{2}\left(\frac{y}{k}\right) \mathrm{d} x \mathrm{~d} y \\
& \leq \frac{\|\eta\|_{\infty}^{2}\left\|\chi^{\prime}\right\|_{\infty}^{2}}{k^{2} \operatorname{vol}(E)} \int_{\mathbb{R}^{2}}\left|\int_{E} g^{\prime}\left(y-\frac{\xi B}{\omega^{2}+B^{2}}\right) e^{i \xi x} \mathrm{~d} \xi\right|^{2} \mathrm{~d} x \mathrm{~d} y \\
& =\frac{\|\eta\|_{\infty}^{2}\left\|\chi^{\prime}\right\|_{\infty}^{2}}{k^{2} \operatorname{vol}(E)} \int_{\mathbb{R}} \int_{E}\left(g^{\prime}\left(y-\frac{x B}{\omega^{2}+B^{2}}\right)\right)^{2} \mathrm{~d} x \mathrm{~d} y \\
& =\frac{\|\eta\|_{\infty}^{2}\left\|\chi^{\prime}\right\|_{\infty}^{2}}{k^{2}} \int_{\mathbb{R}}\left(g^{\prime}\right)^{2}(z) \mathrm{d} z
\end{aligned}
$$


where in the last step we employed again Plancherel formula, and similarly,

$$
\begin{aligned}
& \frac{1}{k^{4} \operatorname{vol}(E)} \int_{\mathbb{R}^{2}}\left|\int_{E} g\left(y-\frac{\xi B}{\omega^{2}+B^{2}}\right) e^{i \xi(x-\alpha k)} \mathrm{d} \xi\right|^{2} \eta^{2}\left(\frac{x}{k}\right)\left(\chi^{\prime \prime}\right)^{2}\left(\frac{y}{k}\right) \mathrm{d} x \mathrm{~d} y \\
& \leq \frac{\|\eta\|_{\infty}^{2}\left\|\chi^{\prime \prime}\right\|_{\infty}^{2}}{k^{4}} \int_{\mathbb{R}} g^{2}(z) \mathrm{d} z \\
& \frac{1}{k^{2} \operatorname{vol}(E)} \int_{\mathbb{R}^{2}}\left|\int_{E} \xi g\left(y-\frac{\xi B}{\omega^{2}+B^{2}}\right) e^{i \xi(x-\alpha k)} \mathrm{d} \xi\right|^{2}\left(\eta^{\prime}\right)^{2}\left(\frac{x}{k}\right) \chi^{2}\left(\frac{y}{k}\right) \mathrm{d} x \mathrm{~d} y \\
& \leq \frac{\left\|\eta^{\prime}\right\|_{\infty}^{2}\|\chi\|_{\infty}^{2} \delta_{2}^{2}(\varepsilon)}{k^{2}} \int_{\mathbb{R}} g^{2}(z) \mathrm{d} z, \\
& \frac{1}{k^{4} \operatorname{vol}(E)} \int_{\mathbb{R}^{2}}\left|\int_{E} g\left(y-\frac{\xi B}{\omega^{2}+B^{2}}\right) e^{i \xi(x-\alpha k)} \mathrm{d} \xi\right|^{2}\left(\eta^{\prime \prime}\right)^{2}\left(\frac{x}{k}\right) \chi^{2}\left(\frac{y}{k}\right) \mathrm{d} x \mathrm{~d} y \\
& \leq \frac{\left\|\eta^{\prime \prime}\right\|_{\infty}^{2}\|\chi\|_{\infty}^{2}}{k^{4}} \int_{\mathbb{R}} g^{2}(z) \mathrm{d} z, \\
& \frac{1}{k^{2} \operatorname{vol}(E)} \int_{\mathbb{R}^{2}} y^{2}\left|\int_{E} g\left(y-\frac{\xi B}{\omega^{2}+B^{2}}\right) e^{i \xi(x-\alpha k)} \mathrm{d} \xi\right|^{2}\left(\eta^{\prime}\right)^{2}\left(\frac{x}{k}\right) \chi^{2}\left(\frac{y}{k}\right) \mathrm{d} x \mathrm{~d} y \\
& \leq \frac{\left\|\eta^{\prime}\right\|_{\infty}^{2}\|\chi\|_{\infty}^{2}}{k^{2}} \int_{\mathbb{R}}\left(|z|+\frac{\delta_{2}(\varepsilon) B}{\omega^{2}+B^{2}}\right)^{2} g^{2}(z) \mathrm{d} z,
\end{aligned}
$$

and consequently, all these integrals are at least $\mathcal{O}\left(k^{-2}\right)$ as $k \rightarrow \infty$. Then we can estimate the norm on left-hand side of (2.5) as

$$
\begin{aligned}
\int_{\mathbb{R}^{2}} & \left|H_{\mathrm{Sm}}(A) \varphi_{k, \alpha, m}-\mu \varphi_{k, \alpha, m}\right|^{2}(x, y) \mathrm{d} x \mathrm{~d} y \\
= & \int_{\mathbb{R}^{2}}\left|-\frac{\partial^{2} \varphi_{k, \alpha, m}}{\partial x^{2}}-\frac{\partial^{2} \varphi_{k, \alpha, m}}{\partial y^{2}}+2 i B y \frac{\partial \varphi_{k, \alpha, m}}{\partial x}+\left(\omega^{2}+B^{2}\right) y^{2} \varphi_{k, \alpha, m}-\mu \varphi_{k, \alpha, m}\right|^{2} \mathrm{~d} x \mathrm{~d} y \\
= & \frac{1}{2 \pi \operatorname{vol}(E)} \int_{\mathbb{R}^{2}} \mid \int_{E}\left(-g^{\prime \prime}\left(y-\frac{\xi B}{\omega^{2}+B^{2}}\right)+\xi^{2} g\left(y-\frac{\xi B}{\omega^{2}+B^{2}}\right)\right. \\
& -2 B y \xi g\left(y-\frac{\xi B}{\omega^{2}+B^{2}}\right)+\left(\omega^{2}+B^{2}\right) y^{2} g\left(y-\frac{\xi B}{\omega^{2}+B^{2}}\right) \\
& \left.-\mu g\left(y-\frac{\xi B}{\omega^{2}+B^{2}}\right)\right)\left.e^{i \xi(x-\alpha k)} \mathrm{d} \xi\right|^{2} \eta^{2}\left(\frac{x}{k}\right) \chi^{2}\left(\frac{y}{k}\right) \mathrm{d} x \mathrm{~d} y+\mathcal{O}\left(k^{-2}\right)
\end{aligned}
$$


A magnetic version of the Smilansky-Solomyak model

$$
\begin{aligned}
= & \frac{1}{2 \pi \operatorname{vol}(E)} \int_{\mathbb{R}^{2}} \mid \int_{E}\left(-g^{\prime \prime}\left(y-\frac{\xi B}{\omega^{2}+B^{2}}\right)+\left(\left(B^{2}+\omega^{2}\right)\left(y-\frac{\xi B}{\omega^{2}+B^{2}}\right)^{2}\right.\right. \\
& \left.\left.+\frac{\omega^{2} \xi^{2}}{\omega^{2}+B^{2}}-\mu\right) g\left(y-\frac{\xi B}{\omega^{2}+B^{2}}\right)\right)\left.e^{i \xi(x-\alpha k)} \mathrm{d} \xi\right|^{2} \eta^{2}\left(\frac{x}{k}\right) \chi^{2}\left(\frac{y}{k}\right) \mathrm{d} x \mathrm{~d} y+\mathcal{O}\left(k^{-2}\right) \\
= & \left.\frac{1}{2 \pi \operatorname{vol}(E)} \int_{\mathbb{R}^{2}} \mid \int_{E}\left(\frac{\omega^{2} \xi^{2}}{\omega^{2}+B^{2}}-\tilde{\mu}\right) g\left(y-\frac{\xi B}{\omega^{2}+B^{2}}\right)\right)\left.e^{i \xi(x-\alpha k)} \mathrm{d} \xi\right|^{2} \\
& \times \eta^{2}\left(\frac{x}{k}\right) \chi^{2}\left(\frac{y}{k}\right) \mathrm{d} x \mathrm{~d} y+\mathcal{O}\left(k^{-2}\right) \\
\leq & \left.\frac{\|\eta\|_{\infty}^{2}\|\chi\|_{\infty}^{2}}{2 \pi \operatorname{vol}(E)} \int_{\mathbb{R}^{2}} \mid \int_{E}\left(\frac{\omega^{2} \xi^{2}}{\omega^{2}+B^{2}}-\tilde{\mu}\right) g\left(y-\frac{\xi B}{\omega^{2}+B^{2}}\right)\right)\left.e^{i \xi x} \mathrm{~d} \xi\right|^{2} \mathrm{~d} x \mathrm{~d} y+\mathcal{O}\left(k^{-2}\right) \\
\leq & \frac{\|\eta\|_{\infty}^{2}\|\chi\|_{\infty}^{2}}{\operatorname{vol}(E)} \int_{E \times \mathbb{R}}\left(\frac{\omega^{2} x^{2}}{\omega^{2}+B^{2}}-\tilde{\mu}\right)^{2} g^{2}\left(y-\frac{x B}{\omega^{2}+B^{2}}\right) \mathrm{d} x \mathrm{~d} y+\mathcal{O}\left(k^{-2}\right) \\
\leq & \frac{\varepsilon^{2}\|\eta\|_{\infty}^{2}\|\chi\|_{\infty}^{2}}{\operatorname{vol}(E)} \int_{E \times \mathbb{R}} g^{2}\left(y-\frac{x B}{\omega^{2}+B^{2}}\right) \mathrm{d} x \mathrm{~d} y+\mathcal{O}\left(k^{-2}\right) \\
\leq & \varepsilon^{2}\|\eta\|_{\infty}^{2}\|\chi\|_{\infty}^{2} \int_{\mathbb{R}} g^{2}(z) \mathrm{d} z+\mathcal{O}\left(k^{-2}\right) .
\end{aligned}
$$

Hence choosing a large enough $k$ we can achieve that

$$
\begin{aligned}
\int_{\mathbb{R}^{2}}\left|H_{\mathrm{Sm}}(A) \varphi_{k, \alpha, m}-\mu \varphi_{k, \alpha, m}\right|^{2}(x, y) \mathrm{d} x \mathrm{~d} y & <64 \varepsilon^{2}\|\eta\|_{\infty}^{2}\|\chi\|_{\infty}^{2} \int_{\mathbb{R}^{2}}\left|\varphi_{k, \alpha, m}\right|^{2} \mathrm{~d} x \mathrm{~d} y+\mathcal{O}\left(k^{-2}\right) \\
& \leq 65 \varepsilon^{2}\|\eta\|_{\infty}^{2}\|\chi\|_{\infty}^{2} \int_{\mathbb{R}^{2}}\left|\varphi_{k, \alpha, m}\right|^{2} \mathrm{~d} x \mathrm{~d} y
\end{aligned}
$$

To complete the proof we choose a sequence $\left\{\varepsilon_{j}\right\}_{j=1}^{\infty}$ such that $\varepsilon_{j} \searrow 0$ holds as $j \rightarrow \infty$ and to any given $j$ we construct a function $\left\{\varphi_{\varepsilon_{j}}\right\}_{j=1}^{\infty}=\left\{\varphi_{k\left(\varepsilon_{j}\right), \alpha\left(k\left(\varepsilon_{j}\right)\right), m\left(\alpha\left(k\left(\varepsilon_{j}\right)\right), k\left(\varepsilon_{j}\right)\right)}\right\}_{j=1}^{\infty}$ with the parameters chosen in such a way that $k\left(\varepsilon_{j}\right)>m\left(\alpha\left(k\left(\varepsilon_{j-1}\right)\right), k\left(\varepsilon_{j-1}\right)\right) k\left(\varepsilon_{j-1}\right)$. The norms of $H_{\mathrm{Sm}}(A) \varphi_{\varepsilon_{j}}$ satisfy the inequality (2.12) with $65 \varepsilon_{j}^{2}\|\eta\|_{\infty}^{2}\|\chi\|_{\infty}^{2}\left\|\varphi_{\varepsilon_{j}}\right\|_{L^{2}\left(\mathbb{R}^{2}\right)}^{2}$ on the right-hand side, and at the same time the sequence converges by construction weakly to zero.

\subsection{Subcritical case: the essential spectrum threshold}

Next we are going to show that in the subcritical case the inclusion in Theorem 2.1 is in fact an equality.

Theorem 2.2. Let $\lambda>-2 \omega$, then the spectrum of $H_{\mathrm{Sm}}(A)$ below $\sqrt{\omega^{2}+B^{2}}$ is purely discrete.

Proof. We employ a Neumann bracketing in a way similar to BE17. Let $h_{n}^{( \pm)}(A)$ and $h_{0}(A)$ be the Neumann restrictions of operator $H_{\mathrm{Sm}}(A)$ to the regions

$$
G_{n}^{( \pm)}=\mathbb{R} \times\{y: \pm y \geq n\}
$$


and $G_{0}=\mathbb{R} \times[-n, n]$, where $n \in \mathbb{N}$ will be chosen later. In view of the minimax principle [RS78, Secs. XIII.1 and XIII.15] we have the inequality

$$
H_{\mathrm{Sm}}(A) \geq\left(h_{n}^{(+)}(A) \oplus h_{n}^{(-)}(A)\right) \oplus h^{(0)}(A) .
$$

To prove the claim we are going first to demonstrate that for a sufficiently large $n$ the spectra of $h_{n}^{( \pm)}(A)$ below $\sqrt{\omega^{2}+B^{2}}$ are empty, and secondly, that for any $\Lambda<\sqrt{\omega^{2}+B^{2}}$ the spectrum of $h_{0}(A)$ below $\Lambda$ is purely discrete.

The quadratic form $Q\left(h_{n}^{( \pm)}(A)\right)$ corresponding to $h_{n}^{( \pm)}(A)$ coincides with

$$
\begin{aligned}
& Q\left(h_{n}^{( \pm)}(A)\right)=\int_{\mathbb{R}} \int_{ \pm y \geq n}\left|\frac{\partial u}{\partial x}-i B y u\right|^{2} \mathrm{~d} x \mathrm{~d} y+\int_{\mathbb{R}} \int_{ \pm y \geq n}\left|\frac{\partial u}{\partial y}\right|^{2} \mathrm{~d} x \mathrm{~d} y \\
& \quad+\omega^{2} \int_{\mathbb{R}} \int_{ \pm y \geq n} y^{2}|u|^{2} \mathrm{~d} x \mathrm{~d} y+\lambda \int_{\mathbb{R}} \int_{ \pm y \geq n} y|u(0, y)|^{2} \mathrm{~d} y \\
& \geq \int_{\mathbb{R}} \int_{ \pm y \geq n}\left|\frac{\partial u}{\partial x}-i B y u\right|^{2} \mathrm{~d} x \mathrm{~d} y+\omega^{2} \int_{\mathbb{R}} \int_{ \pm y \geq n} y^{2}|u|^{2} \mathrm{~d} x \mathrm{~d} y+\lambda \int_{\mathbb{R}} \int_{ \pm y \geq n} y|u(0, y)|^{2} \mathrm{~d} y
\end{aligned}
$$

defined on $\mathcal{H}^{1}(\mathbb{R} \times\{y: \pm y \geq n\})$. As before the quadratic forms

$$
u(\cdot, y) \mapsto \int_{\mathbb{R}}\left|i \frac{\partial u}{\partial x}-B y u\right|^{2}+\omega^{2} y^{2}|u|^{2} \mathrm{~d} x+\lambda y|u(0, y)|^{2}
$$

are for any fixed $y$ unitarily equivalent to $y^{2} L$ if $y>0$ and $y^{2} \widetilde{L}$ if $y<0$, where $\widetilde{L}$ is given in (2.2). Thus we have

$$
\begin{aligned}
& \inf \sigma\left(h_{n}^{ \pm}\right) \geq n^{2} \inf \sigma(L)=n^{2}\left(\omega^{2}-\frac{\lambda^{2}}{4}\right) \quad \text { if } \quad y>0, \\
& \inf \sigma\left(h_{n}^{ \pm}\right) \geq n^{2} \inf \sigma(\widetilde{L})=n^{2} ; \omega^{2} \quad \text { if } \quad y<0
\end{aligned}
$$

recall that we suppose $\lambda<0$. This concludes the proof of our first claim provided one chooses a sufficiently large $n$. It remains to inspect the essential spectrum of $h_{0}(A)$. To this aim we employ the following auxiliary result.

Lemma 2.1. Under our assumptions

$$
\inf \sigma_{\text {ess }}\left(h_{0}(A)\right)=\inf \sigma_{\text {ess }}\left(\tilde{h}_{0}(A)\right)
$$

where $\tilde{h}_{0}(A)$ is the Neumann operator $\left(i \frac{\partial}{\partial x}-B y\right)^{2}-\frac{\partial^{2}}{\partial y^{2}}+\omega^{2} y^{2}$ defined on $\mathcal{H}^{1}\left(G_{0}\right)$.

Proof. We are going to show that for any real number $\mu$ from the resolvent sets of both $h_{0}(A)$ and $\tilde{h}_{0}(A)$ the operator

$$
W:=\left(h_{0}(A)-\mu \mathbb{I}\right)^{-1}-\left(\tilde{h}_{0}(A)-\mu \mathbb{I}\right)^{-1}
$$


is compact in $L^{2}\left(G_{0}\right)$. We proceed as in [BEL13] for any fixed $f, g \in L^{2}\left(G_{0}\right)$ we put $u:=\left(h_{0}(A)-\mu \mathbb{I}\right)^{-1} f$ and $v:=\left(\tilde{h}_{0}(A)-\mu \mathbb{I}\right)^{-1} g$. Then

$$
\begin{aligned}
& (W f, g)=\left(\left(h_{0}(A)-\mu \mathbb{I}\right)^{-1} f, g\right)-\left(\left(\tilde{h}_{0}(A)-\mu \mathbb{I}\right)^{-1} f, g\right) \\
& \quad=(u, g)-(f, v)=\left(u,\left(h_{0}(A)-\mu \mathbb{I}\right) v\right)-\left(\left(h_{0}(A)-\mu \mathbb{I}\right) u, v\right) \\
& \quad=\left(\tilde{h}_{0}(A) u, v\right)-\left(h_{0}(A) u, v\right)=-\alpha \int_{-n}^{n} y u(0, y) \overline{v(0, y)} \mathrm{d} y .
\end{aligned}
$$

Let $\left\{f_{n}\right\}_{n=1}^{\infty}$ and $\left\{g_{n}\right\}_{n=1}^{\infty}$ be bounded sequences in $L^{2}\left(G_{0}\right)$. Then it follows from the Sobolev trace theorem [M00] that the $\mathcal{H}^{1}\left(G_{0}\right)$ bounded functions $u_{n}:=$ $\left(h_{0}(A)-\mu \mathbb{I}\right)^{-1} f_{n}$ and $v_{n}=\left(\tilde{h}_{0}(A)-\mu \mathbb{I}\right)^{-1} g_{n}$ are also bounded in $\mathcal{H}^{1 / 2}(x=0,|y| \leq n)$. In view of the compact embedding $\mathcal{H}^{1 / 2}(x=0,|y| \leq n) \hookrightarrow L^{2}(x=0,|y| \leq n)$ the sequences $\left\{u_{n}\right\}$ and $\left\{v_{n}\right\}_{n=1}^{\infty}$ are compact in $L^{2}(x=0,|y| \leq n)$. In combination with the inequality

$$
\begin{aligned}
& \left(W\left(f_{n}-f_{m}\right), W\left(f_{n}-f_{m}\right)\right) \\
& \quad \leq|\alpha| n \sqrt{\int_{-n}^{n}\left|\left(u_{n}-u_{m}\right)(0, y)\right|^{2} \mathrm{~d} y} \sqrt{\int_{-n}^{n}\left|\left(\tilde{v}_{n}-\tilde{v}_{m}\right)(0, y)\right|^{2} \mathrm{~d} y}
\end{aligned}
$$

with $\tilde{v}_{n}=\left(\tilde{h}_{0}(A)-\mu \mathbb{I}\right)^{-1}\left(W\left(f_{n}-f_{m}\right)\right)$, this establishes our claim.

Let us now return to the proof of the theorem. In view of the lemma it is sufficient to inspect the threshold of $\sigma_{\text {ess }}\left(\tilde{h}_{0}(A)\right)$. Using the partial Fourier transformation $\mathrm{F}_{\xi}(u(x, y))$ given by

$$
\mathrm{F}_{\xi}(u(x, y))=\widehat{u}(x, y)=\frac{1}{\sqrt{2 \pi}} \int_{\mathbb{R}} u(\xi, y) e^{-i \xi x} \mathrm{~d} \xi
$$

and the Landau gauge for the vector potential, $A=(-B y, 0)$, one is able to rewrite the quadratic form $Q_{0}$ of $\tilde{h}_{0}(A)$ as

$$
\begin{aligned}
& \left.Q_{0}(u)=\left.\int_{\mathbb{R} \times[-n, n]}(\mid i \nabla u+A u)\right|^{2}(x, y)+\omega^{2} y^{2}|u|^{2}(x, y)\right) \mathrm{d} x \mathrm{~d} y \\
& =\int_{\mathbb{R} \times[-n, n]}\left[\left|\frac{\partial \widehat{u}}{\partial y}\right|^{2}(\xi, y)+\left(\left(\omega^{2}+B^{2}\right)\left(y-\frac{\xi B}{\omega^{2}+B^{2}}\right)^{2}+\frac{\omega^{2} \xi^{2}}{\omega^{2}+B^{2}}\right)|\widehat{u}|^{2}(\xi, y)\right] \mathrm{d} \xi \mathrm{d} y .
\end{aligned}
$$

Thus $\tilde{h}_{0}(A)$ is unitarily equivalent to the direct integral

$$
\int_{\mathbb{R}}^{\oplus} h(\xi) \mathrm{d} \xi,
$$

where the fibers $h(\xi)=-\frac{\mathrm{d}^{2}}{\mathrm{~d} y^{2}}+\left(\omega^{2}+B^{2}\right)\left(y-\frac{\xi B}{\omega^{2}+B^{2}}\right)^{2}+\frac{\omega^{2} \xi^{2}}{\omega^{2}+B^{2}}$ are one-dimensional Neumann operators defined on $L^{2}(-n, n)$. By a simple change of variables we arrive at the Neumann harmonic oscillators $l(\xi)=-\frac{\mathrm{d}^{2}}{\mathrm{~d} y^{2}}+\left(\omega^{2}+B^{2}\right) y^{2}+\frac{\omega^{2} \xi^{2}}{\omega^{2}+B^{2}}$ on the interval $\left[-n-\frac{\xi B}{\omega^{2}+B^{2}}, n-\frac{\xi B}{\omega^{2}+B^{2}}\right]$. Similarly as in [BE17] one can check that

$$
\inf \sigma(l(\xi)) \geq \sqrt{\omega^{2}+B^{2}}+\mathcal{O}\left(n^{-1}\right)
$$


holds for large $n$ uniformly in $\xi \in \mathbb{R}$. The spectrum of $\sigma\left(\tilde{h}_{0}(A)\right)$ is determined by those of the fiber operators [RS78, Sec. XIII.16], in particular we get

$$
\sigma\left(\tilde{h}_{0}(A)\right)=\sigma_{\text {ess }}\left(\tilde{h}_{0}(A)\right) \subset\left[\sqrt{\omega^{2}+B^{2}}+\mathcal{O}\left(n^{-1}\right), \infty\right) .
$$

By virtue of Lemma 2.1 this yields

$$
\inf \sigma_{\text {ess }}\left(h_{0}(A)\right) \geq \sqrt{\omega^{2}+B^{2}}+\mathcal{O}\left(n^{-1}\right),
$$

and therefore for any $\Lambda<\sqrt{\omega^{2}+B^{2}}$ one can choose $n$ large enough to ensure that the spectrum of $h_{0}(A)$ below $\Lambda$ is purely discrete which concludes the proof.

\subsection{Subcritical case: existence of the discrete spectrum}

The above results localize exactly the essential spectrum, however, they tell us nothing about the existence of the discrete spectrum. This is the question we are going to address now.

Theorem 2.3. Let $\lambda>-2 \omega$, then the discrete spectrum of $H_{\mathrm{Sm}}(A)$ is nonempty and contained in the interval $\left(0, \sqrt{\omega^{2}+B^{2}}\right)$.

Proof. To demonstrate the non-emptiness of the discrete spectrum one needs to construct a normalized function $u \in \operatorname{Dom}\left(Q\left(H_{\mathrm{Sm}}(A)\right)\right)$ such that $Q\left(H_{\mathrm{Sm}}(A)\right)(u)<$ $\sqrt{\omega^{2}+B^{2}}$. On the other hand, since $\lambda>-2 \omega>-2 \sqrt{\omega^{2}+B^{2}}$ the non-magnetic operator $\widetilde{H}=-\Delta+\left(\omega^{2}+B^{2}\right) y^{2}+\lambda y \delta(x)$ has a nonempty finite set of eigenvalues below $\sqrt{\omega^{2}+B^{2}}$, and moreover, the corresponding eigenfunctions can be chosen realvalued So04b]. It is easy to see that for any such eigenfunction $u$ we have

$$
Q(H(A))(u)=Q(\widetilde{H})(u)<\sqrt{\omega^{2}+B^{2}},
$$

and since by Proposition 2.1 operator $H_{\mathrm{Sm}}(A)$ is positive, the claimed is proved.

\subsection{The supercritical case}

Let us now turn to the situation when the coupling constant surpasses the critical value. As discussed in the opening of Sec. 2, we know that operator $H_{\mathrm{Sm}}(A) \uparrow \mathcal{D}_{0}$ has self-adjoint extensions and in the following we will use the symbol $H_{\mathrm{Sm}(A)}$ for any of them.

Theorem 2.4. $\sigma\left(H_{\mathrm{Sm}}(A)\right)=\mathbb{R}$ holds provided $\lambda<-2 \omega$.

Proof. To check that any real number $\mu$ belongs to the spectrum of $H_{\mathrm{Sm}}(A)$ we employ Weyl's criterion finding a sequence $\left\{\psi_{k}\right\}_{k=1}^{\infty} \subset D\left(H_{\mathrm{Sm}}(A)\right)$ such that $\left\|\psi_{k}\right\|=1$ satisfying

$$
\left\|H_{\mathrm{Sm}}(A) \psi_{k}-\mu \psi_{k}\right\| \rightarrow 0 \quad \text { as } \quad k \rightarrow \infty ;
$$

note that one need not require that $\left\{\psi_{k}\right\}$ contains no convergent subsequence because the spectrum covering the whole real axis cannot be anything else than essential. To this 
aim we modify the method of [BE14] without repetition of the parts that do not change. With scaling transformations in mind we may suppose that inf $\sigma(L)=\omega^{2}-\frac{1}{4} \lambda^{2}=-1$ corresponding to the single eigenvalue of the operator which is simple and associated with the normalized eigenfunction $h=\sqrt{\frac{|\lambda|}{2}} e^{-|\lambda||t| / 2}$.

As in BE14 we will first show that $0 \in \sigma\left(H_{\mathrm{Sm}}(A)\right)$. We fix an $\varepsilon>0$ and choose a positive integer $k=k(\varepsilon)$ to which we associate a function $\chi_{k} \subset C_{0}^{2}(1, k)$ such that

$$
\int_{1}^{k} \frac{1}{z} \chi_{k}^{2}(z) \mathrm{d} z=1 \quad \text { and } \quad \int_{1}^{k} z\left(\chi_{k}^{\prime}(z)\right)^{2} \mathrm{~d} z<\varepsilon ;
$$

we know from [BE14] that such functions can be constructed. Then we define

$$
\psi_{k}(x, y):=h(x y) \mathrm{e}^{i y^{2} / 2} \chi_{k}\left(\frac{y}{n_{k}}\right)+\frac{f(x y)}{y^{2}} \mathrm{e}^{i y^{2} / 2} \chi_{k}\left(\frac{y}{n_{k}}\right),
$$

where the smooth function $f \in \operatorname{Dom}(L)$ and the positive integer $n_{k} \in \mathbb{N}$ will be chosen later. The functions (2.19) belong to $\operatorname{Dom}\left(H_{\mathrm{Sm}}(A)\right)$ by construction and have the following property [BE14].

Lemma 2.2. $\left\|\psi_{k}\right\|_{L^{2}\left(\mathbb{R}^{2}\right)} \geq \frac{1}{2}$ holds provided $n_{k}$ is large enough.

Next we have to show that the functions $\psi_{k}$ approximate the generalized eigenfunction corresponding to zero energy.

Lemma 2.3. $\left\|H_{\mathrm{Sm}}(A) \psi_{k}\right\|_{L^{2}\left(\mathbb{R}^{2}\right)}^{2}<c \varepsilon$ holds with a $c$ independent of $k$ provided $n_{k}$ is large enough.

Proof. We have to estimate the following integral,

$\int_{\mathbb{R}^{2}}\left|H_{\mathrm{Sm}}(A) \psi_{k}\right|^{2}(x, y) \mathrm{d} x \mathrm{~d} y=\int_{\mathbb{R}^{2}}\left|-\frac{\partial^{2} \psi_{k}}{\partial x^{2}}-\frac{\partial^{2} \psi_{k}}{\partial y^{2}}+2 i B x \frac{\partial \psi_{k}}{\partial y}+B^{2} x^{2} \psi_{k}+\omega^{2} y^{2} \psi_{k}\right|^{2} \mathrm{~d} x \mathrm{~d} y$.

We know from [BE14] that the claim is valid if $B=0$, hence it remains to deal with the additional terms associated with the magnetic field. We have

$$
\begin{aligned}
\frac{\partial \psi_{k}}{\partial y}= & \left(x h^{\prime}(x y) \chi_{k}\left(\frac{y}{n_{k}}\right)+\frac{1}{n_{k}} h(x y) \chi_{k}^{\prime}\left(\frac{y}{n_{k}}\right)+\frac{x}{y^{2}} f^{\prime}(x y) \chi_{k}\left(\frac{y}{n_{k}}\right)+\frac{i}{y} f(x y) \chi_{k}\left(\frac{y}{n_{k}}\right)\right. \\
& \left.+\frac{1}{n_{k} y^{2}} f(x y) \chi_{k}^{\prime}\left(\frac{y}{n_{k}}\right)-\frac{2}{y^{3}} f(x y) \chi_{k}\left(\frac{y}{n_{k}}\right)+i y h(x y) \chi_{k}\left(\frac{y}{n_{k}}\right)\right) \mathrm{e}^{i y^{2} / 2}
\end{aligned}
$$

which allows us to check that choosing $n_{k}$ large enough one can make norms of all the 
A magnetic version of the Smilansky-Solomyak model

terms in the expression of $x \frac{\partial \psi_{k}}{\partial y}$ except of the last one small enough, because

$$
\begin{aligned}
\int_{\mathbb{R}^{2}}\left|x^{2} h^{\prime}(x y) \mathrm{e}^{i y^{2} / 2} \chi_{k}\left(\frac{y}{n_{k}}\right)\right|^{2} \mathrm{~d} x \mathrm{~d} y & \leq \frac{1}{n_{k}^{4}} \int_{\mathbb{R}}\left|h^{\prime}(t)\right|^{2} \mathrm{~d} t \int_{1}^{k}\left|\chi_{k}(z)\right|^{2} \mathrm{~d} z, \\
\frac{1}{n_{k}^{2}} \int_{\mathbb{R}^{2}}\left|h(x y) \mathrm{e}^{i y^{2} / 2} \chi_{k}^{\prime}\left(\frac{y}{n_{k}}\right)\right|^{2} \mathrm{~d} x \mathrm{~d} y & \leq \frac{1}{n_{k}^{2}} \int_{\mathbb{R}}|h(t)|^{2} \mathrm{~d} t \int_{1}^{k}\left|\chi_{k}^{\prime}(z)\right|^{2} \mathrm{~d} z, \\
\int_{\mathbb{R}^{2}}\left|\frac{x}{y^{2}} f^{\prime}(x y) \mathrm{e}^{i y^{2} / 2} \chi_{k}\left(\frac{y}{n_{k}}\right)\right|^{2} \mathrm{~d} x \mathrm{~d} y & \leq \frac{1}{n_{k}^{4}} \int_{\mathbb{R}}\left|f^{\prime}(t)\right|^{2} \mathrm{~d} t \int_{1}^{k}\left|\chi_{k}(z)\right|^{2} \mathrm{~d} z, \\
\int_{\mathbb{R}^{2}}\left|\frac{i}{y} f(x y) \mathrm{e}^{i y^{2} / 2} \chi_{k}\left(\frac{y}{n_{k}}\right)\right|^{2} \mathrm{~d} x \mathrm{~d} y & \leq \frac{1}{n_{k}^{2}} \int_{\mathbb{R}}|f(t)|^{2} \mathrm{~d} t \int_{1}^{k}\left|\chi_{k}(z)\right|^{2} \mathrm{~d} z, \\
\int_{\mathbb{R}^{2}}\left|\frac{1}{n_{k} y^{2}} f(x y) \mathrm{e}^{i y^{2} / 2} \chi_{k}^{\prime}\left(\frac{y}{n_{k}}\right)\right|^{2} \mathrm{~d} x \mathrm{~d} y & \leq \frac{1}{n_{k}^{6}} \int_{\mathbb{R}}|f(t)|^{2} \mathrm{~d} t \int_{1}^{k}\left|\chi_{k}^{\prime}(z)\right|^{2} \mathrm{~d} z, \\
\int_{\mathbb{R}^{2}}\left|\frac{2}{y^{3}} f(x y) \mathrm{e}^{i y^{2} / 2} \chi_{k}\left(\frac{y}{n_{k}}\right)\right|^{2} \mathrm{~d} x \mathrm{~d} y & \leq \frac{4}{n_{k}^{6}} \int_{\mathbb{R}}|f(t)|^{2} \mathrm{~d} t \int_{1}^{k}\left|\chi_{k}(z)\right|^{2} \mathrm{~d} z .
\end{aligned}
$$

In a similar way one can check that for large $n_{k}$ the integral $\int_{\mathbb{R}^{2}}\left|x^{2} \psi_{k}\right|^{2} \mathrm{~d} x \mathrm{~d} y$ is less than $\varepsilon$. This yields the estimate

$$
\begin{gathered}
\int_{\mathbb{R}^{2}}\left|H_{\mathrm{Sm}}(A) \psi_{k}\right|^{2}(x, y) \mathrm{d} x \mathrm{~d} y \leq 21 \int_{n_{k}}^{k n_{k}} \int_{\mathbb{R}} \mid y^{2}\left(h^{\prime \prime}(x y)-\omega^{2} h(x y)-h(x y)\right) \chi_{k}\left(\frac{y}{n_{k}}\right) \\
\quad+i h(x y) \chi_{k}\left(\frac{y}{n_{k}}\right)+f^{\prime \prime}(x y) \chi_{k}\left(\frac{y}{n_{k}}\right)+2 i x y h^{\prime}(x y) \chi_{k}\left(\frac{y}{n_{k}}\right)+\frac{2 i y}{n_{k}} h(x y) \chi_{k}^{\prime}\left(\frac{y}{n_{k}}\right) \\
\quad-f(x y) \chi_{k}\left(\frac{y}{n_{k}}\right)-\omega^{2} f(x y) \chi_{k}\left(\frac{y}{n_{k}}\right)+\left.2 \operatorname{Bxyh}(x y) \chi_{k}\left(\frac{y}{n_{k}}\right)\right|^{2} \mathrm{~d} x \mathrm{~d} y+21 \varepsilon,
\end{gathered}
$$

where the coefficient in front of the integrals comes from the number of the summands. Using the fact that $L h=-h$ and applying the Cauchy inequality, the above inequality implies

$$
\begin{aligned}
\frac{1}{21} \int_{\mathbb{R}^{2}}\left|H_{\mathrm{Sm}}(A) \psi_{k}\right|^{2}(x, y) \mathrm{d} x \mathrm{~d} y<\int_{n_{k}}^{k n_{k}} \int_{\mathbb{R}} \mid\left(f^{\prime \prime}(x y)+2 i x y h^{\prime}(x y)+i h(x y)-f(x y)\right. \\
\left.\quad-\omega^{2} f(x y)+2 B x y h(x y)\right) \chi_{k}\left(\frac{y}{n_{k}}\right)+\left.\frac{2 i y}{n_{k}} h(x y) \chi_{k}^{\prime}\left(\frac{y}{n_{k}}\right)\right|^{2} \mathrm{~d} x \mathrm{~d} y+\varepsilon \\
\left.\leq 2 \int_{1}^{k} \frac{1}{z}\left|\chi_{k}(z)\right|^{2} \mathrm{~d} z \int_{\mathbb{R}} \mid-f^{\prime \prime}(t)+f(t)\left(1+\omega^{2}\right)\right)-2 i t h^{\prime}(t) \\
\quad-i h(t)-\left.2 B t h(t)\right|^{2} \mathrm{~d} t+8 \int_{1}^{k} z\left|\chi_{k}^{\prime}(z)\right|^{2} \mathrm{~d} z+\varepsilon \\
\leq 2 \int_{\mathbb{R}}\left|-f^{\prime \prime}(t)+f(t)\left(1+\omega^{2}\right)-2 i t h^{\prime}(t)-i h(t)-2 B t h(t)\right|^{2} \mathrm{~d} t+9 \varepsilon
\end{aligned}
$$

It is easy to check that

$$
\int_{\mathbb{R}}\left(2 i t h^{\prime}(t)+i h(t)+2 B t h(t)\right) h(t) \mathrm{d} t=0,
$$


which together with the simplicity of the eigenvalue -1 establishes the existence of the solution for the differential equation

$$
-f^{\prime \prime}(t)+f(t)\left(1+\omega^{2}\right)-2 i t h^{\prime}(t)-i h(t)-2 B t h(t)=0
$$

belonging to $\operatorname{Dom}(L)$ which will use in the Ansatz (2.19). With this choice the last integral in the above estimate vanishes, which gives

$$
\int_{\mathbb{R}^{2}}\left|H_{\mathrm{Sm}}(A) \psi_{k}\right|^{2}(x, y) \mathrm{d} x \mathrm{~d} y \leq 189 \varepsilon
$$

concluding this the proof of the lemma.

Now we can complete the proof of the theorem. We fix a sequence $\left\{\varepsilon_{j}\right\}_{j=1}^{\infty}$ such that $\varepsilon_{j} \searrow 0$ holds as $j \rightarrow \infty$ and to any $j$ we construct a function $\psi_{k\left(\varepsilon_{j}\right)}$. As we have mentioned it is not necessary that such a sequence converges weakly to zero, but we can achieve that at no extra expense by choosing the corresponding numbers in such a way that $n_{k\left(\varepsilon_{j}\right)}>k\left(\varepsilon_{j-1}\right) n_{k\left(\varepsilon_{j-1}\right)}$. The norms of $H_{\mathrm{Sm}}(A) \psi_{k\left(\varepsilon_{j}\right)}$ satisfy inequality which (2.21) with $189 \varepsilon_{j}$ on the right-hand side, which yields the desired result.

For any nonzero real number $\mu$ we use the same procedure replacing (2.19) with

$$
\psi_{k}(x, y)=h(x y) \mathrm{e}^{i \epsilon_{\mu}(y)} \chi_{k}\left(\frac{y}{n_{k}}\right)+\frac{f(x y)}{y^{2}} \mathrm{e}^{i \epsilon_{\mu}(y)} \chi_{k}\left(\frac{y}{n_{k}}\right),
$$

where $\epsilon_{\mu}(y):=\int_{\sqrt{|\mu|}}^{y} \sqrt{t^{2}+\mu} \mathrm{d} t$ and the functions $f, \chi_{k}$ are the same as above. Repeating the estimates with the modified exponential function one can check that to any positive $\varepsilon$ one can choose the integer $n_{k}$ large enough so that

$$
\begin{aligned}
\| \frac{\partial^{2} \psi_{k}}{\partial y^{2}} \mathrm{e}^{-i \varepsilon_{\mu}(y)}-2 i B x \frac{\partial \psi_{k}}{\partial y} \mathrm{e}^{-i \varepsilon_{\mu}(y)}+\mu \psi_{k} \mathrm{e}^{-i \varepsilon_{\mu}(y)} \\
\quad-\mathrm{e}^{-i y^{2} / 2}\left(\frac{\partial^{2}}{\partial y^{2}}\left(\psi_{k} \mathrm{e}^{-i \varepsilon_{\mu}(y)+i y^{2} / 2}\right)-2 i B x \frac{\partial}{\partial y}\left(\psi_{k} \mathrm{e}^{-i \varepsilon_{\mu}(y)+i y^{2} / 2}\right)\right) \|_{L^{2}\left(\mathbb{R}^{2}\right)}<\varepsilon
\end{aligned}
$$

holds. Using further the identity $\frac{\partial^{2} \psi_{k}}{\partial x^{2}} \mathrm{e}^{-i \varepsilon_{\mu}(y)}=\mathrm{e}^{-i y^{2} / 2} \frac{\partial^{2}}{\partial x^{2}}\left(\psi_{k} \mathrm{e}^{-i \varepsilon_{\mu}(y)+i y^{2} / 2}\right)$ we get

$$
\begin{gathered}
\left\|H_{\mathrm{Sm}}(A) \psi_{k}-\mu \psi_{k}\right\|_{L^{2}\left(\mathbb{R}^{2}\right)}=\left\|\left(H_{\mathrm{Sm}}(A) \psi_{k}\right) \mathrm{e}^{-i \varepsilon_{\mu}(y)}-\mu \psi_{k} \mathrm{e}^{-i \varepsilon_{\mu}(y)}\right\|_{L^{2}\left(\mathbb{R}^{2}\right)} \\
<\left\|\mathrm{e}^{-i y^{2} / 2} H_{\mathrm{Sm}}(A)\left(\psi_{k} \mathrm{e}^{-i \varepsilon_{\mu}(y)+i y^{2} / 2}\right)\right\|_{L^{2}\left(\mathbb{R}^{2}\right)}+\varepsilon
\end{gathered}
$$

now we can use the result of the first part of proof to conclude the proof.

Remark 2.1. In view of Theorem 2.1 we could have restricted our attention to the numbers $\mu<\sqrt{\omega^{2}+B^{2}}$ only. Avoiding this restriction makes sense, however, showing that in the supercritical case one can construct for any $\mu$ a Weyl sequence with the support in the vicinity of the $y$ axis. Looking at the problem from the dynamical point of view as in Gu11, this fact is connected with the existence of states escaping to infinity along the singular channel. 


\subsection{The critical case}

If the two competing forces are in exact balance, $\lambda=-2 \omega$, the quadratic form is still positive by Proposition 2.1 , hence $H_{\mathrm{Sm}}(A)$ can be defined as the Friedrich's extension of the operator initially defined on set $\mathcal{D}_{0}$.

Theorem 2.5. Let $\lambda=-2 \omega$, then under the stated assumptions we have

$$
\sigma\left(H_{\mathrm{Sm}}(A)\right)=\sigma_{\mathrm{ess}}\left(H_{\mathrm{Sm}}\right)=[0, \infty) .
$$

Proof. In view of Proposition 2.1 it is enough to show that $\sigma_{\mathrm{ess}}\left(H_{\mathrm{Sm}}\right)(A) \supset[0, \infty)$. We proceed as in the previous section: to any $\mu \geq 0$ we are again going to construct a sequence $\left\{\psi_{n}\right\}_{n=1}^{\infty} \subset D\left(H_{\mathrm{Sm}}(A)\right)$ of unit vectors, $\left\|\psi_{n}\right\|=1$, such that

$$
\left\|H_{\mathrm{Sm}}(A) \psi_{n}-\mu \psi_{n}\right\| \rightarrow 0 \quad \text { as } \quad n \rightarrow \infty .
$$

holds. The operator $L_{0}=-\frac{\mathrm{d}^{2}}{\mathrm{~d} x^{2}}+\lambda \delta(x)$ on $L^{2}(\mathbb{R})$ has a single eigenvalue equal to $-\frac{1}{4} \lambda^{2}$, hence its spectral threshold of is an isolated eigenvalue corresponding to the normalized eigenfunction $h$, the same as in the previous section. Given a smooth function $\chi$ with $\operatorname{supp} \chi \subset[1,2]$ and satisfying $\int_{1}^{2} \chi^{2}(z) \mathrm{d} z=1$, we put

$$
\psi_{n}(x, y):=h(x y) \mathrm{e}^{i \sqrt{\mu} y} \chi\left(\frac{y}{n}\right)
$$

where $n \in \mathbb{N}$ is to be chosen later. For the moment we just note that choosing $n$ large enough one can achieve that $\left\|\psi_{n}\right\|_{L^{2}\left(\mathbb{R}^{2}\right)} \geq \frac{1}{\sqrt{2}}$ as the following estimates show,

$$
\begin{aligned}
& \int_{\mathbb{R}^{2}}\left|h(x y) \mathrm{e}^{i \sqrt{\mu} y} \chi\left(\frac{y}{n}\right)\right|^{2} \mathrm{~d} x \mathrm{~d} y=\int_{n}^{2 n} \int_{\mathbb{R}}\left|h(x y) \chi\left(\frac{y}{n}\right)\right|^{2} \mathrm{~d} x \mathrm{~d} y \\
& =\int_{n}^{2 n} \int_{\mathbb{R}} \frac{1}{y}\left|h(t) \chi\left(\frac{y}{n}\right)\right|^{2} \mathrm{~d} t \mathrm{~d} y=\int_{n}^{2 n} \frac{1}{y}\left|\chi\left(\frac{y}{n}\right)\right|^{2} \mathrm{~d} y=\int_{1}^{2} \frac{1}{z}|\chi(z)|^{2} \mathrm{~d} z \geq \frac{1}{2} .
\end{aligned}
$$

Next we are going to show that $\left\|H_{\mathrm{Sm}}(A) \psi_{n}-\mu \psi_{n}\right\|_{L^{2}\left(\mathbb{R}^{2}\right)}^{2}<\varepsilon$ holds for a suitably chosen $n=n(\varepsilon)$. By a straightforward computation we express $\frac{\partial^{2} \psi_{n}}{\partial x^{2}}, \frac{\partial^{2} \psi_{n}}{\partial y^{2}}$, and $x \frac{\partial \psi_{n}}{\partial y}$; in the same way as in the previous section one can check that the norms of the last two can be made as small as we wish by choosing $n$ sufficiently large. Moreover, we have

$$
\int_{\mathbb{R}^{2}}\left|x^{2} h(x y) \mathrm{e}^{i \sqrt{\mu} y} \chi\left(\frac{y}{n}\right)\right|^{2} \mathrm{~d} x \mathrm{~d} y \leq \frac{1}{n^{4}} \int_{1}^{2} \frac{|\chi(z)|^{2}}{z} \mathrm{~d} z \int_{\mathbb{R}}|h(t)|^{2} \mathrm{~d} t,
$$

hence this term too can be made small. This allows us to estimate the expression in question as

$$
\begin{aligned}
\int_{\mathbb{R}^{2}} & \left|H_{\mathrm{Sm}}(A) \psi_{n}-\mu \psi_{n}\right|^{2}(x, y) \mathrm{d} x \mathrm{~d} y \\
= & \int_{\mathbb{R}^{2}}\left|-\frac{\partial^{2} \psi_{n}}{\partial x^{2}}-\frac{\partial^{2} \psi_{n}}{\partial y^{2}}+2 i B x \frac{\partial \psi_{n}}{\partial y}+B^{2} x^{2} \psi_{n}+\omega^{2} y^{2} \psi_{n}-\mu \psi_{n}\right|^{2} \mathrm{~d} x \mathrm{~d} y \\
= & \int_{n}^{2 n} \int_{\mathbb{R}}\left|y^{2}\left(-h^{\prime \prime}(x y)+\omega^{2} h(x y)\right) \chi\left(\frac{y}{n}\right)\right|^{2} \mathrm{~d} x \mathrm{~d} y+\varepsilon
\end{aligned}
$$


for all sufficiently large $n$, and using the fact that $L h=0$ holds by assumption, we get from here

$$
\int_{\mathbb{R}^{2}}\left|H_{\mathrm{Sm}}(A) \psi_{n}-\mu \psi_{n}\right|^{2}(x, y) \mathrm{d} x \mathrm{~d} y<\varepsilon,
$$

which is what we have set out to demonstrate.

\section{Spectrum of $H(A)$}

Now we pass to the 'regular' version of the magnetic Smilansky-Solomyak model described by the Hamiltonian (1.4). As before, the first question to address concerns its self-adjointness. In this case we can check that $H(A)$ is essentially self-adjoint on $C_{0}^{\infty}\left(\mathbb{R}^{2}\right)$ with a reference to [190]: it is sufficient to find a sequence of non-overlapping annular regions $A_{m}=\left\{z \in \mathbb{R}^{2}: a_{m}<|z|<b_{m}\right\}$ and a sequence of positive numbers $\nu_{m}$ such that

$$
\left(b_{m}-a_{m}\right)^{2} \nu_{m}>K, \quad V(z) \geq-k \nu_{m}^{2}\left(b_{m}-a_{m}\right)^{2} \quad \text { for } z \in A_{m} \quad \text { and } \quad \sum_{m=1}^{\infty} \nu_{m}^{-1}=\infty
$$

where $K$ and $k$ are positive constants independent of $m$. It can be seen easily that for $a_{m}=m, b_{m}=m+1$, and $\nu_{m}=m+1, m=0,1,2, \ldots$, the requirement (3.1) is satisfied if we choose $K=\frac{1}{2}$ and $k=|\lambda|\|V\|_{\infty}$.

\subsection{Subcritical case: positivity and essential spectrum}

As before we show first that the operator is positive in the subcritical situation.

Proposition 3.1. $H(A) \geq 0$ holds provided inf $\sigma(L(V)) \geq 0$.

Proof. The argument is mimicking the reasoning used in Proposition 2.1. For any $u \in \operatorname{Dom}(Q(H(A))) \subset \mathcal{H}^{1}\left(\mathbb{R}^{2}\right)$ one has

$$
\begin{aligned}
& Q(H(A))(u)=\int_{\mathbb{R}^{2}}\left|i \frac{\partial u}{\partial x}-B y u\right|^{2}+\left|\frac{\partial u}{\partial y}\right|^{2}+\left(\omega^{2} y^{2}+\lambda y^{2} V(x y)\right)|u|^{2} \mathrm{~d} x \mathrm{~d} y \\
& \geq \int_{\mathbb{R}^{2}}\left|i \frac{\partial u}{\partial x}-B y u\right|^{2}+\left(\omega^{2} y^{2}+\lambda y^{2} V(x y)\right)|u|^{2} \mathrm{~d} x \mathrm{~d} y .
\end{aligned}
$$

Furthermore, the quadratic forms

$$
u(\cdot, y) \mapsto \int_{\mathbb{R}}\left|i \frac{\partial u}{\partial x}-B y u\right|^{2}+\left(\omega^{2} y^{2}+\lambda y^{2} V(x y)\right)|u|^{2} \mathrm{~d} x
$$

with a fixed $y$ correspond to the operators

$$
\left(i \frac{\mathrm{d}}{\mathrm{d} x}-B y\right)^{2}+\omega^{2} y^{2}+\lambda y^{2} V(x y) \text { on } \mathcal{H}^{1}(\mathbb{R})
$$

which are unitarily equivalent to $y^{2} L(V) \geq 0$. 
As in the case of $H_{\mathrm{Sm}}(A)$ the 'unperturbed' essential spectrum is preserved independently of the value the coupling constant $\lambda$ may take.

Theorem 3.1. $\sigma_{\mathrm{ess}}(H(A)) \supset\left[\sqrt{\omega^{2}+B^{2}}, \infty\right)$.

Proof. As before we have to construct a Weyl sequence for any $\mu \geq \sqrt{\omega^{2}+B^{2}}$, in other words, to find to any $\varepsilon>0$ a function $\phi$ such that

$$
\|H(A) \phi-\mu \phi\|_{L^{2}\left(\mathbb{R}^{2}\right)}<\varepsilon\|\phi\|
$$

We employ the functions defined by (2.6) which obviously belong to the domain of $H(A)$. The only change on the right-hand side of (2.5) comes now from the addition of the term $\lambda y^{2} V(x y) \varphi_{k, \alpha, m}$. Using the fact that $V$ is by assumption compactly supported, we infer that

$$
\begin{aligned}
& \frac{1}{2 \pi \operatorname{vol}(E)} \int_{\mathbb{R}^{2}} y^{2}\left|\int_{E} g\left(y-\frac{\xi B}{\omega^{2}+B^{2}}\right) e^{i \xi(x-\alpha k)} \mathrm{d} \xi\right|^{2} V^{2}(x y) \eta^{2}\left(\frac{x}{k}\right) \chi^{2}\left(\frac{y}{k}\right) \mathrm{d} x \mathrm{~d} y \\
& \leq \frac{\|\eta\|_{\infty}^{2}\|\chi\|_{\infty}^{2}\|V\|_{\infty}^{2}}{\operatorname{vol}(E)} \int_{E \times\left\{|y| \leq \frac{s_{0}}{k}\right\}} y^{2} g^{2}\left(y-\frac{x B}{\omega^{2}+B^{2}}\right) \mathrm{d} x \mathrm{~d} y \\
& \leq \frac{s_{0}^{2}\|\eta\|_{\infty}^{2}\|\chi\|_{\infty}^{2}\|V\|_{\infty}^{2}}{k^{2} \operatorname{vol}(E)} \int_{E \times \mathbb{R}} g^{2}\left(y-\frac{x B}{\omega^{2}+B^{2}}\right) \mathrm{d} x \mathrm{~d} y \\
& =\frac{s_{0}^{2}\|\eta\|_{\infty}^{2}\|\chi\|_{\infty}^{2}\|V\|_{\infty}^{2}}{k^{2}} \int_{\mathbb{R}} g^{2}(z) \mathrm{d} z .
\end{aligned}
$$

Consequently, choosing $k$ large enough one can achieve that the above integral will be sufficiently small, which together with the inequality (2.12) implies the validity of 3.2 . The rest of the argument is the same as in Theorem 2.1.

\subsection{Subcritical case: essential spectrum threshold}

While the value of $\lambda$ was irrelevant in the previous theorem, it becomes important if we ask about the essential spectrum threshold.

Theorem 3.2. Let inf $\sigma(L(V))>0$, then the spectrum of $H(A)$ below $\sqrt{\omega^{2}+B^{2}}$ is purely discrete.

Proof. We employ Neumann bracketing combined with the minimax principle in a way similar to that used in [BE17]. By $h_{n}^{( \pm)}(A, V)$ and $h_{0}(A, V)$ we denote the Neumann restrictions of operator $H(A)$ to the strips

$$
G_{n}^{( \pm)}=\mathbb{R} \times\{y: 1+\ln n< \pm y \leq 1+\ln (n+1)\}, n \geq n_{0},
$$

and $G_{0}=\mathbb{R} \times\left[-1-\ln n_{0}, 1+\ln n_{0}\right]$, where $n_{0} \in \mathbb{N}$ will be chosen later. It allows us to estimated the operator from below,

$$
H(A) \geq\left(\bigoplus_{n=n_{0}}^{\infty} h_{n}^{(+)}(A, V) \oplus h_{n}^{(-)}(A, V)\right) \oplus h_{0}(A, V)
$$


To prove the result we have to show first that the spectral thresholds of $h_{n}^{( \pm)}(A, V)$ tend to infinity as $n \rightarrow \infty$, and secondly, that for any $\Lambda<\sqrt{\omega^{2}+B^{2}}$ one can choose $n_{0}$ in such a way that the spectrum of $h_{0}(A, V)$ below $\Lambda$ is purely discrete. The function $V$ is by assumption compactly supported with a bounded derivative, hence we have

$$
V(x y)-V(x(1+\ln n))=\mathcal{O}\left(\frac{1}{n \ln n}\right), \quad y^{2}-(1+\ln n)^{2}=\mathcal{O}\left(\frac{\ln n}{n}\right)
$$

for any $(x, y) \in G_{n}^{(+)}$and an analogous relation for $G_{n}^{(-)}$, and consequently,

$$
y^{2} V(x y)-(1+\ln n)^{2} V( \pm x(1+\ln n))=\mathcal{O}\left(\frac{\ln n}{n}\right)
$$

holds for any $(x, y) \in G_{n}^{( \pm)}$. Moreover, for any function $u \in \mathcal{H}^{1}\left(G_{n}\right)$ and any fixed positive $\varepsilon$ we have

$$
\begin{aligned}
& \int_{G_{n}}\left|i \frac{\partial u}{\partial x}-B y u\right|^{2} \mathrm{~d} x \mathrm{~d} y=\int_{G_{n}}\left|i \frac{\partial u}{\partial x}-B \ln n u+B(\ln n-y) u\right|^{2} \mathrm{~d} x \mathrm{~d} y \\
& \quad \geq \int_{G_{n}}\left|i \frac{\partial u}{\partial x}-B \ln n u\right|^{2} \mathrm{~d} x \mathrm{~d} y-2 \int_{G_{n}} \sqrt{\varepsilon}\left|i \frac{\partial u}{\partial x}-B \ln n u\right| \frac{B}{\sqrt{\varepsilon}}|\ln n-y||u| \mathrm{d} x \mathrm{~d} y \\
& \quad \geq(1-\varepsilon) \int_{G_{n}}\left|i \frac{\partial u}{\partial x}-B \ln n u\right|^{2} \mathrm{~d} x \mathrm{~d} y-\frac{B^{2}}{\varepsilon} \int_{G_{n}}(\ln (n+1)-\ln n)^{2}|u|^{2} \mathrm{~d} x \mathrm{~d} y,
\end{aligned}
$$

and therefore

$$
\left(i \frac{\partial}{\partial x}-B y\right)^{2} \geq(1-\varepsilon)\left(i \frac{\partial}{\partial x}-B \ln n\right)^{2}-\frac{B^{2}}{\varepsilon}(\ln (n+1)-\ln n)^{2},
$$

which together with (3.4) implies the asymptotic inequalities

$$
\inf \sigma\left(h_{n}^{( \pm)}(A, V)\right) \geq(1-\varepsilon) \inf \sigma\left(l_{n}^{( \pm)}(A, V)\right)+\mathcal{O}\left(\frac{\ln n}{n}\right),
$$

in which the operators

$l_{n}^{( \pm)}(A, V):=\left(i \frac{\partial}{\partial x}-B \ln n\right)^{2}-\frac{\partial^{2}}{\partial y^{2}}+\omega^{2}(1+\ln n)^{2}+\frac{\lambda}{1-\varepsilon}(1+\ln n)^{2} V( \pm x(1+\ln n))$

with Neumann conditions are defined on $G_{n}^{( \pm)}$. Since $l_{n}^{ \pm}(A, V)$ is easily seen to be unitarily equivalent to the non-magnetic operator $\tilde{l}_{n}^{ \pm}(V)=-\frac{\partial^{2}}{\partial x^{2}}-\frac{\partial^{2}}{\partial y^{2}}+\omega^{2}(1+\ln n)^{2}+$ $\frac{\lambda}{1-\varepsilon}(1+\ln n)^{2} V( \pm x(1+\ln n))$ defined on the same domain, their spectra coincide. The operator $\tilde{l}_{n}^{ \pm}(V)$ allows the separation of variables. Since the principal eigenvalue of $-\frac{\mathrm{d}^{2}}{\mathrm{~d} y^{2}}$ on an any interval with Neumann boundary conditions is zero, we have

$$
\inf \sigma\left(\tilde{l}_{n}^{ \pm}(V)\right)=\inf \sigma\left(l_{n}(V)\right),
$$

where $l_{n}(V)=-\frac{\mathrm{d}^{2}}{\mathrm{~d} x^{2}}+\omega^{2}(1+\ln n)^{2}+\frac{\lambda}{1-\varepsilon}(1+\ln n)^{2} V( \pm x(1+\ln n))$. By the change of variable, $x=\frac{t}{1+\ln n}$, the last named operator is in turn unitarily equivalent to 
$(1+\ln n)^{2} L_{\varepsilon}(V)$ with $L_{\varepsilon}(V)=\frac{\mathrm{d}^{2}}{\mathrm{~d} t^{2}}+\omega^{2}+\frac{\lambda}{1-\varepsilon} V$, and therefore in view of inequality (3.6) the relation $\inf \sigma\left(\tilde{l}_{n}^{ \pm}(V)\right)=(1+\ln n)^{2} \inf \sigma\left(L_{\varepsilon}(V)\right)$ holds, which together with the first order of perturbation-theory argument and (3.5) concludes the proof of the discreteness of $\bigoplus_{n=1}^{\infty} h_{n}^{(+)}(A, V) \oplus h_{n}^{(-)}(A, V)$.

It remains to inspect the spectrum of $h_{0}(A, V)$. To proceed with the proof we need the following auxiliary result.

Lemma 3.1. Under our assumptions

$$
\inf \sigma_{\text {ess }}\left(h_{0}(A, V)\right)=\inf \sigma_{\text {ess }}\left(\tilde{h}_{0}(A)\right),
$$

where $\tilde{h}_{0}(A)$ is the operator $\left(i \frac{\partial}{\partial x}-B y\right)^{2}-\frac{\partial^{2}}{\partial y^{2}}+\omega^{2} y^{2}$ on $L^{2}\left(G_{0}\right)$ with Neumann boundary conditions.

Proof. From the minimax principle [RS78, Secs. XIII.1 and XIII.15] it follows that

$$
\inf \sigma_{\text {ess }}\left(h_{0}(A, V)\right) \leq \inf \sigma_{\text {ess }}\left(\tilde{h}_{0}(A)\right) .
$$

To establish the opposite inequality it is enough to check that the spectrum of $h_{0}(A, V)$ is purely discrete below $\inf \sigma_{\text {ess }}\left(\tilde{h}_{0}(A)\right)$. Given a $k \in \mathbb{N}$, we introduce the operator $h_{1}(A, V)=\left(i \frac{\partial}{\partial x}-B y\right)^{2}-\frac{\partial^{2}}{\partial x^{2}}+\omega^{2} y^{2}+\lambda y^{2} V(x y) \chi_{\{|x| \leq k\}}(x)$ for some large $k \in \mathbb{N}$. It differs from $h_{0}(A, V)$ by the potential term in the region $\{|x|>k\} \times \mathbb{R}$, however, the potential $V$ is compactly supported by assumption, and therefore only $y \in\left(-\frac{s_{0}}{k}, \frac{s_{0}}{k}\right)$ must be considered and we get

$$
h_{0}(A, V) \geq h_{1}(A, V)-\frac{s_{0}^{2}|\lambda|\|V\|_{\infty}}{k^{2}},
$$

hence $\inf \sigma_{\text {ess }}\left(h_{0}(A, V)\right) \geq \inf \sigma_{\text {ess }}\left(h_{1}(A, V)\right)+\mathcal{O}\left(k^{-2}\right)$. Since $k$ can be chosen arbitrarily large, the identity (3.8) would follow if we check that

$$
\sigma_{\text {ess }}\left(h_{1}(A, V)\right)=\sigma_{\text {ess }}\left(\tilde{h}_{0}(A)\right) .
$$

To this aim we use the stability of the essential spectrum against compact perturbations [RS78, Sec. XIII.4], specifically, we check the compactness of the resolvent difference $\left(h_{1}(A, V)-z \mathbb{I}\right)^{-1}-\left(\tilde{h}_{0}(A)-z \mathbb{I}\right)^{-1}$ as an operator on $L^{2}\left(G_{0}\right)$ for $z$ belonging to both the resolvent sets of $h_{1}(A, V)$ and $\tilde{h}_{0}(A)$. Using the resolvent identity we write the difference in question as

$$
\left(h_{1}(A, V)-z \mathbb{I}\right)^{-1}\left(h_{1}(A, V)-\tilde{h}_{0}(A)\right)\left(\tilde{h}_{0}(A)-z \mathbb{I}\right)^{-1} .
$$

It is easy to realize that for any bounded $\mathcal{U} \subset L^{2}\left(G_{0}\right)$ the set $\left(\tilde{h}_{0}(A)-z \mathbb{I}\right)^{-1} \mathcal{U}$ is uniformly $\mathcal{H}^{1}$ bounded. Furthermore, $h_{1}(A, V)-\tilde{h}_{0}(A)$ is by construction a compactly supported potential in $\{|x| \leq k\} \times\left\{|y| \leq n_{0}\right\}$, which implies its boundedness in $H^{1}(\Omega)$, where $\Omega=$ $\left.\{|x| \leq k\} \times\left\{|y| \leq n_{0}\right\}\right) \cap\{(x, y): x y \in \operatorname{supp} V\}$. Finally, using the embedding theorems for Sobolev spaces on bounded domains we conclude that $\left(h_{1}(A, V)-\tilde{h}_{0}(A)\right) \mathcal{V}$ is compact in $L^{2}(\Omega)$ which implies the same also for $\left(h_{1}(A, V)-z \mathbb{I}\right)^{-1}\left(\left(h_{1}(A, V)-\tilde{h}_{0}(A)\right) \mathcal{V}\right)$, and thus the claim we have set out to prove.

The rest is simple: combining the preceding lemma with the inclusion (2.17) we verify the claim of Theorem 3.2. 


\subsection{Subcritical case: existence of the discrete spectrum}

As in the previous section, proving that the spectrum below $\sqrt{\omega^{2}+B^{2}}$ ) says nothing about its existence, it has to be checked separately.

Theorem 3.3. Let $\inf \sigma(L(V))>0$, then the discrete spectrum of $H(A)$ is non-empty and contained in the interval $\left(0, \sqrt{\omega^{2}+B^{2}}\right)$.

Proof. We have to construct a normalized trial function $\phi$ such that the corresponding value of the quadratic form $Q(H(A))$ will be less than $\sqrt{\omega^{2}+B^{2}}$. This time we employ the letter $h$ to denote the normalized ground-state eigenfunction of the one-dimensional harmonic oscillator, $h_{\mathrm{osc}}=-\frac{\mathrm{d}^{2}}{\mathrm{~d} y^{2}}+\left(\omega^{2}+B^{2}\right) y^{2}$ on $L^{2}(\mathbb{R})$, and set

$$
\phi(x, y):=\frac{1}{\sqrt{k}} h(y) \chi\left(\frac{x}{k}\right)
$$

where $\chi(z)$ is a real-valued smooth function with $\operatorname{supp}(\chi)=[-1,1]$ such that

$$
\int_{-1}^{1} \chi^{2}(z) \mathrm{d} z=1, \quad \min _{|z| \leq 1 / 2} \chi(z)=: \alpha>0,
$$

and $k$ is a natural number to be chosen later. A straightforward computation yields

$$
\begin{aligned}
Q(H(A))[\phi]= & \int_{\mathbb{R}^{2}}\left|\frac{\partial \phi}{\partial x}\right|^{2} \mathrm{~d} x \mathrm{~d} y+\int_{\mathbb{R}^{2}}\left|\frac{\partial \phi}{\partial y}\right|^{2} \mathrm{~d} x \mathrm{~d} y+\int_{\mathbb{R}^{2}}\left(\omega^{2}+B^{2}\right) y^{2}|\phi|^{2} \mathrm{~d} x \mathrm{~d} y \\
& +\lambda \int_{\mathbb{R}^{2}} y^{2} V(x y)\left|\phi^{2}\right| \mathrm{d} x \mathrm{~d} y
\end{aligned}
$$

because the contribution from the terms containing the first derivatives is easily seen to vanish, and therefore

$$
\begin{aligned}
& Q(H(A))[\phi]=\frac{1}{k^{3}} \int_{\mathbb{R}^{2}} h^{2}(y)\left(\chi^{\prime}\right)^{2}\left(\frac{x}{k}\right) \mathrm{d} x \mathrm{~d} y+\frac{1}{k} \int_{\mathbb{R}^{2}}\left(h^{\prime}\right)^{2}(y) \chi^{2}\left(\frac{x}{k}\right) \mathrm{d} x \mathrm{~d} y \\
& \quad+\frac{1}{k} \int_{\mathbb{R}^{2}}\left(\omega^{2}+B^{2}\right) y^{2} h^{2}(y) \chi^{2}\left(\frac{x}{k}\right) \mathrm{d} x \mathrm{~d} y+\frac{\lambda}{k} \int_{\mathbb{R}^{2}} y^{2} V(x y) h^{2}(y) \chi^{2}\left(\frac{x}{k}\right) \mathrm{d} x \mathrm{~d} y \\
& =\mathcal{O}\left(k^{-2}\right)+\frac{1}{k} \int_{\mathbb{R}^{2}}\left(\left(h^{\prime}\right)^{2}(y)+\left(\omega^{2}+B^{2}\right) y^{2} h^{2}(y)\right) \chi^{2}\left(\frac{x}{k}\right) \mathrm{d} x \mathrm{~d} y \\
& \quad+\frac{\lambda}{k} \int_{\mathbb{R}^{2}} y^{2} V(x y) h^{2}(y) \chi^{2}\left(\frac{x}{k}\right) \mathrm{d} x \mathrm{~d} y \\
& =\mathcal{O}\left(k^{-2}\right)+\frac{\sqrt{\omega^{2}+B^{2}}}{k} \int_{\mathbb{R}^{2}} h^{2}(y) \chi^{2}\left(\frac{x}{k}\right) \mathrm{d} x \mathrm{~d} y+\frac{\lambda}{k} \int_{\mathbb{R}^{2}} y^{2} V(x y) h^{2}(y) \chi^{2}\left(\frac{x}{k}\right) \mathrm{d} x \mathrm{~d} y \\
& =\mathcal{O}\left(k^{-2}\right)+\sqrt{\omega^{2}+B^{2}}+\frac{\lambda}{k} \int_{\mathbb{R}^{2}} y^{2} V(x y) h^{2}(y) \chi^{2}\left(\frac{x}{k}\right) \mathrm{d} x \mathrm{~d} y .
\end{aligned}
$$


We need to estimate the last term on the right-hand side of (3.10). One has

$$
\begin{aligned}
& \frac{|\lambda|}{k} \int_{\mathbb{R}^{2}} y^{2} V(x y) h^{2}(y) \chi^{2}\left(\frac{x}{k}\right) \mathrm{d} x \mathrm{~d} y=\frac{|\lambda|}{k} \int_{-k}^{k} \int_{\mathbb{R}} y^{2} V(x y) h^{2}(y) \chi^{2}\left(\frac{x}{k}\right) \mathrm{d} x \mathrm{~d} y \\
& \geq \frac{|\lambda|}{k} \int_{-k / 2}^{k / 2} \int_{0}^{\infty} y^{2} V(x y) h^{2}(y) \chi^{2}\left(\frac{x}{k}\right) \mathrm{d} x \mathrm{~d} y \geq \frac{\alpha^{2}|\lambda|}{k} \int_{-k / 2}^{k / 2} \int_{0}^{\infty} y^{2} V(x y) h^{2}(y) \mathrm{d} x \mathrm{~d} y \\
& =\frac{\alpha^{2} \lambda}{k} \int_{0}^{\infty} \int_{-k y / 2}^{k y / 2} y V(t) h^{2}(y) \mathrm{d} t \mathrm{~d} y \geq \frac{\alpha^{2}|\lambda|}{k} \int_{1}^{\infty} \int_{-k / 2}^{k / 2} y V(t) h^{2}(y) \mathrm{d} t \mathrm{~d} y \\
& \geq \frac{\alpha^{2}|\lambda|}{k} \int_{1}^{\infty} y h^{2}(y) \mathrm{d} y \int_{-k / 2}^{k / 2} V(t) \mathrm{d} t .
\end{aligned}
$$

If $k$ is chosen large enough the above estimate implies

$$
\frac{|\lambda|}{k} \int_{\mathbb{R}^{2}} y^{2} V(x y) h^{2}(y) \chi^{2}\left(\frac{x}{k}\right) \mathrm{d} x \mathrm{~d} y \geq \frac{\alpha^{2}|\lambda|}{k} \int_{1}^{\infty} y h^{2}(y) \mathrm{d} y \int_{-s_{0}}^{s_{0}} V(t) \mathrm{d} t,
$$

hence in combination with (3.10) we infer that

$$
Q(H(A))[\phi] \leq \mathcal{O}\left(k^{-2}\right)+\sqrt{\omega^{2}+B^{2}}-\frac{\alpha^{2}|\lambda|}{k} \int_{1}^{\infty} y h^{2}(y) \mathrm{d} y \int_{-a}^{a} V(t) \mathrm{d} t,
$$

where the right-hand side is obviously less than $\sqrt{\omega^{2}+B^{2}}$ for all $k$ large enough.

\subsection{The supercritical and critical cases}

Let us turn next to the case where the 'escape to infinity' is possible.

Theorem 3.4. Under our hypotheses, assuming in addition that the potential $V$ is symmetric with respect to the origin, $\sigma(H(A))=\mathbb{R}$ holds if $\inf \sigma(L(V))<0$.

The proof is completely the same as in Theorem 2.4, the only difference concerns the substitution of the function $h$ into the normalized eigenfunction of $L(V)$. The symmetry of the potential $V$ is needed to guarantee the existence of the solutions of the differential equation (2.20).

Finally, in the critical case we have

Theorem 3.5. $\sigma(H(A))=\sigma_{\text {ess }}(H(A))=[0, \infty)$ holds provided $\inf \sigma(L(V))=0$.

The proof repeats the almost exactly the argument leading to Theorem 2.5.

\section{Acknowledgements}

The authors are grateful to V. Lotoreichik for a useful discussion. The research has been supported by the Czech Science Foundation (GAČR) within the project 17-01706S. D.B. also acknowledges a support from the projects 01211/2016/RRC and the Czech-Austrian Grant CZ 02/2017. 


\section{References}

[AGHH05] Albeverio S, Gesztesy F, Høegh-Krohn R and Holden H 2005 Solvable Models in Quantum Mechanics, 2nd edition (Providence, R.I.: AMS Chelsea Publishing)

[BE14] Barseghyan D and Exner P 2014 A regular version of Smilansky model, J. Math. Phys. 55 042104

[BE17] Barseghyan D and Exner P 2017 A regular analogue of the Smilansky model: spectral properties, Rep. Math. Phys., to appear; arXiv:1609.03008

[BEKT16] Barseghyan D, Exner P, Khrabustovskyi A and Tater M 2016 Spectral analysis of a class of Schrödinger operators exhibiting a parameter-dependent spectral transition, J. Phys. A: Math. Theor. 49, 165302

[BEL13] Behrndt J, Exner P and Lotoreichik V 2013 Essential Spectrum of Schrödinger operators with $\delta$ interactions on the union of compact Lipschitz hypersurfaces, Proc. Appl. Math. Mech. 13, $523-524$

[CFKS87] Cycon HL, Froese RG, Kirsch W and Simon B 1987 Schrödinger Operators, with Applications to Quantum Mechanics and Global Geometry (Berlin and Heidelberg: Springer)

[ES05a] Evans WD and Solomyak MZ 2005 Smilanskys model of irreversible quantum graphs. I: The absolutely continuous spectrum, J. Phys. A: Math. Gen. 38, 4611-4627

[ES05b] Evans WD and Solomyak MZ 2005 Smilanskys model of irreversible quantum graphs. II: The point spectrum, J. Phys. A: Math. Gen. 38, 7661-7675

[EB12] Exner P and Barseghyan D 2012 Spectral estimates for a class of Schrödinger operators with infinite phase space and potential unbounded from below, J. Phys. A: Math. Theor. 45, 075204

[EK00] Exner P and Kovařík H 2000 Magnetic strip waveguides, J. Phys. A: Math. Gen. 33 (2000), $3297-3311$

[EK15] Exner P and Kovařík H 2015 Quantum Waveguides (Cham: Springer).

[ELT17] Exner P, Lotoreichik V and Tater M 2017 Spectral and resonance properties of Smilansky Hamiltonian, Phys. Lett. A 381, 756-761

[FGW00] Fröhlich J, Graf GM and Walcher J 2000 On the extended nature of edge states of quantum Hall Hamiltonians, Ann. Henri Poincaré 1 (2000), 405-442

[GMNT16] Gesztesy F, Mitrea M, Nenciu I, Teschl G 2016 Decoupling of deficiency indices and applications to Schrödinger-type operators with possibly strongly singular potentials, Adv. Math. 301, 1022-1061

[Gu11] Guarneri I 2011 Irreversible behaviour and collapse of wave packets in a quantum system with point interactions, J. Phys. A: Math. Theor. 44, 485304

[190] Iwatsuka A 1990 Essential self-adjointness of the Schrödinger operators with magnetic fields diverging at infinity, Publ. RIMS 26, 841-860.

[M00] McLean W 2000 Strongly Elliptic Systems and Boundary Integral Equations, (Cambridge University Press)

[NS06] Naboko S and Solomyak M 2006 On the absolutely continuous spectrum in a model of an irreversible quantum graph, Proc. Lond. Math. Soc. 9, 251-272

[RS80] Reed M and Simon B 1980 Methods of Modern Mathematical Physics, I. Functional Analysis 2nd edition (New York: Academic Press)

[RS78] Reed M and Simon B 1978 Methods of Modern Mathematical Physics, IV. Analysis of Operators (New York: Academic Press)

[RS07] Rozenblum G and Solomyak M 2007 On a family of differential operators with the coupling parameter in the boundary condition, J. Comput. Appl. Math. 208, 57-71

[Sm04] Smilansky U 2004 Irreversible quantum graphs Waves Random Media 14, 143-153

[So04a] Solomyak MZ 2004 On a differential operator appearing in the theory of irreversible quantum graphs, Waves Random Media 14, 173-185

[So04b] Solomyak MZ 2004 On the discrete spectrum of a family of differential operators, Funct. Anal. Appl. 38, 217-223.

[So06a] Solomyak M 2006 On a mathematical model of irreversible quantum graphs, St. Petersburg 
Math. J. $17835-864$

[So06b] Solomyak M 2006 On the limiting behaviour of the spectra of a family of differential operators, J. Phys. A: Math. Gen. 39, 10477-10489

[Zn98] Znojil M 1998 Quantum exotic: a repulsive and bottomless confining potential, J.Phys. A: Math. Gen. 31, 3349-3355 